\title{
Neuregulin-1 enhances cell-cycle activity, delays cardiac fibrosis, and improves cardiac performance in rat pups with right ventricular pressure load
}

\author{
Guido P. L. Bossers, MD, ${ }^{a}$ Marie Günthel, Dipl-Ing, ${ }^{\mathrm{b}}$ Diederik E. van der Feen, MD, ${ }^{\mathrm{a}}$
}

Quint A. J. Hagdorn, MD, PhD, ${ }^{a}$ Anne-Marie C. Koop, MD, PhD, ${ }^{a}$ Karel van Duijvenboden, $\mathrm{PhD},{ }^{\mathrm{b}}$

Phil Barnett, PhD, ${ }^{b}$ Marinus A. J. Borgdorff, MD, PhD, ${ }^{a}$ Vincent M. Christoffels, $\mathrm{PhD},{ }^{b}$

Herman H. W. Silljé, PhD, ${ }^{\mathrm{c}}$ Rolf M. F. Berger, MD, PhD, ${ }^{\mathrm{a}}$ and Beatrijs Bartelds, $\mathrm{MD}, \mathrm{PhD}^{\mathrm{a}}$

\section{ABSTRACT}

Objectives: Right ventricular (RV) failure is a leading cause of death in patients with congenital heart disease. RV failure is kept at bay during childhood. Limited proliferation of cardiomyocytes is present in the postnatal heart. We propose that cardiomyocyte proliferation improves RV adaptation to pressure load (PL). We studied adaptation in response to increased RV PL and the role of increased cardiomyocyte cell cycle activity (CCA) in rat pups growing into adulthood.

Methods: We induced RV PL at day of weaning in rats (3 weeks; $30-40$ g) by pulmonary artery banding and followed rats into adulthood (300 g). We performed histological analyses and RNA sequencing analysis. To study the effects of increased cardiomyocyte cell cycle activity, we administered neuregulin-1 (NRG1), a growth factor involved in cardiac development.

Results: PL induced an increase in CCA, with subsequent decline of CCA (sham/PL at 4 weeks: $0.14 \% / 0.83 \% ; P=.04$ and 8 weeks: $0.00 \% / 0.00 \% ; P=.484$ ) and cardiac function (cardiac index: control/PL 4 weeks: $4.41 / 3.29 ; P=.468$ and 8 weeks: 3.57/1.44; $P=.024$ ). RNA sequencing analysis revealed delayed maturation and increased CCA pathways. NRG1 stimulated CCA (PL vehicle/NRG1 at 2 weeks: $0.62 \% / 2.28 \% ; P=.003$ ), improved cardiac function (cardiac index control vs vehicle/NRG1 at 2 weeks: 4.21 vs 3.07/4.17; $P=.009 / .705)$ and postponed fibrosis (control vs vehicle/NRG1 at 4 weeks: 1.66 vs $4.82 \% / 2.97 \%$; $P=.009 / .078$ ) in RV $\mathrm{PL}$ rats during childhood.

Conclusions: RV PL during growth induces a transient CCA increase. Further CCA stimulation improves cardiac function and delays fibrosis. This proof-of-concept study shows that stimulation of CCA can improve RV adaptation to PL in the postnatal developing heart and might provide a new approach to preserve RV function in patients with congenital heart disease. (J Thorac Cardiovasc Surg 2022;164:e493510)

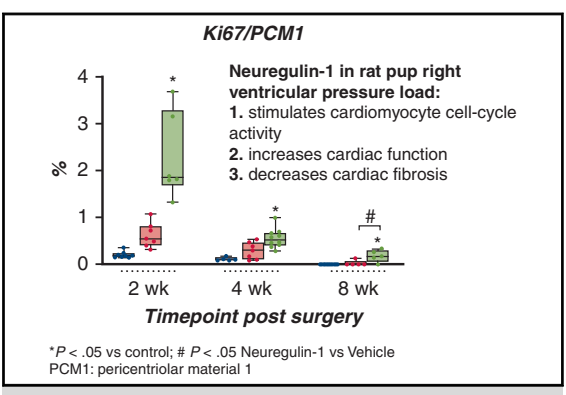

Enhanced cell cycle activity improves outcome in childhood right ventricle pressure load.

\section{CENTRAL MESSAGE}

Neuregulin-1 stimulated cell cycle activity, postponed fibrosis, and improved cardiac function and outcome during childhood, suggesting a new approach to preserve the RV in patients with congenital heart disease.

\section{PERSPECTIVE}

Right ventricular (RV) function and failure are key determinants of morbidity and mortality in congenital heart disease and pulmonary hypertension. No treatment options are available for these patients. Interestingly, RV failure due to chronic pressure overload commences mainly after adulthood is reached. Putatively, processes of adaptation may prevent the RV from failing during childhood.

See Commentary on page e511.

\footnotetext{
From the ${ }^{\mathrm{a} C e n t e r}$ for Congenital Heart Diseases, Department of Pediatric Cardiology, and ${ }^{\mathrm{c}}$ Department of Cardiology, University of Groningen, University Medical Center Groningen, Groningen, The Netherlands; and ${ }^{\mathrm{b}}$ Department of Medical Biology, Amsterdam Cardiovascular Sciences, Amsterdam UMC, Amsterdam, The Netherlands.

This work was supported by a grant from the Dutch Heart Foundation (NHS2013T091), Stichting Hartekind, and the Sebald Fund. B.B. and R.M.F.B. acknowledge the support from The Netherlands CardioVascular Research Initiative: the Dutch Heart Foundation, Dutch Federation of University Medical Centers, The Netherlands Organisation for Health Research and Development, and the Royal Netherlands Academy of Sciences (CVON-2012-08).
}

Current address of Dr B. Bartelds: Department of Pediatrics, Division of Cardiology, Erasmus University Medical Centre-Sophia Children's Hospital, Rotterdam, The Netherlands.

Received for publication Jan 29, 2021; revisions received Oct 11, 2021; accepted for publication Oct 19, 2021; available ahead of print Nov 3, 2021.

Address for reprints: Guido P. L. Bossers, MD, University Medical Center Groningen, Hanzeplein 1, 9700RB Groningen, The Netherlands (E-mail: g.p.l.bossers@ umcg.nl).

$0022-5223$

Copyright (C) 2021 The Authors. Published by Elsevier Inc. on behalf of The American Association for Thoracic Surgery. This is an open access article under the CC BY license (http://creativecommons.org/licenses/by/4.0/).

https://doi.org/10.1016/j.jtcvs.2021.10.045 


$$
\begin{aligned}
& \text { Abbreviations and Acronyms } \\
& \begin{aligned}
\text { CCA } & =\text { cardiomyocyte cell cycle activity } \\
\text { CHD } & =\text { congenital heart disease } \\
\text { H3P } & =\text { histone- } 3 \text {-phosphate } \\
\text { Myh6 } & =\text { myosin heavy chain } 6 \\
\text { Myh7 } & =\text { myosin heavy chain } 7 \\
\text { NRG1 } & =\text { neuregulin- } \\
\text { PCM-1 } & =\text { pericentriolar material } 1 \\
\text { PL } & =\text { pressure load } \\
\text { RV } & =\text { right ventricular }
\end{aligned}
\end{aligned}
$$

Video clip is available online.

Right ventricular (RV) dysfunction is a leading cause of heart failure and death in patients with congenital heart disease (CHD), heart failure with preserved ejection fraction, and pulmonary arterial hypertension. ${ }^{1}$ Increased pressure load (PL) is an important determinant in the development of RV failure in CHD (eg, pulmonary hypertension, hypoplastic left heart syndrome). ${ }^{2}$ The detection and treatment of RV failure recently has been issued as one of the 3 major research focuses by the National Heart, Lung, and Blood Institute and the Adult Congenital Heart Association working group.

The adaptation of a normal morphological RV to chronic PL has been studied extensively in adult patients with pulmonary hypertension and experimental models mimicking PL induced mainly in adult animals. ${ }^{4}$ These studies have shown that the development of RV failure is characterized by progressive diastolic dysfunction, RVpulmonary arterial uncoupling, myocardial fibrosis, and metabolic dysregulation. ${ }^{5,6}$ Unfortunately, treatment options for the failing right ventricle have proven to be disappointing. ${ }^{4,7}$

In patients with severe CHD, RV failure typically develops in the second or third decade. ${ }^{8}$ It is yet unknown what protective mechanisms help children with CHD to sustain increased PL throughout growth and development.

The cardiomyocyte has a high proliferative potential before birth, which, in the period (mouse: days; human: years) after birth virtually disappears. ${ }^{9,10}$ It is yet unknown how this residual proliferative potential after birth affects the adaptive response to CHD-associated RV PL. Various pathways have been identified that can stimulate cardiomyocyte cell cycle activity (CCA). Exploration of these pathways might help identify new therapeutic strategies. ${ }^{11}$ Neuregulin-1 (NRG1) is a growth factor that acts through the v-erb-b2 erythroblastic leukemia viral oncogene homolog signaling pathway. NRG1-mediated signaling is involved in cardiac development and can increase CCA. ${ }^{12}$ It is unknown whether stimulation of CCA in the postnatal growing and developing myocardium under stress is cardioprotective and might be able to postpone the development of heart failure.

The aim of the present study was to assess the adaptation to PL of the right ventricle in the postnatal developing heart growth and development and to test the effects of therapeutic stimulation with NRG1 on cardiac function. For that purpose, we have developed a model of chronic PL of the right ventricle in rat pups, starting at the age of weaning toward young adulthood (body weight from $30 \mathrm{~g}$ to approximately $300 \mathrm{~g}$ ), and studied $\mathrm{RV}$ hemodynamic adaptation and remodeling. Young adulthood in humans correlates with 9-12 weeks after birth in rats. ${ }^{13}$ In this model, we tested in vivo the effects of NRG1 administration on CCA and cardiac performance.

\section{METHODS}

Animal care and experiments were conducted according to the Dutch Animal Experimental Act and conform to the Guide for the Care and Use of Laboratory Animals published by the US National Institutes of Health (publication 85-23, revised 1996). The Animal Experiments Committee of the University of Groningen in the Netherlands approved the experimental protocol (permit number: AVD10500201515134; date of approval: July 29, 2015).

\section{Animal Model}

Figure 1, A, shows the schematic outline of the experiment. Wistar rat pups (male; Charles River) were randomized after weaning (third week of life; 30-40 g) into 2 groups: sham-operated rats (control, $\mathrm{n}=13$ ) and rats with a pulmonary artery banding (PL, $\mathrm{n}=22$ ). One animal died during PL surgery and was excluded from the study. Pulmonary artery banding was applied with the animals under general anesthesia with ventilation (isoflurane/air mixture, $5 \%$ induction; $2 \%-3 \%$ maintenance; analgesia with carprofen $5 \mathrm{mg} / \mathrm{kg}$ subcutaneous) via a left thoracotomy as described previously. ${ }^{5}$ After a 22 -gauge needle was placed in line with the pulmonary artery, a suture around the pulmonary artery and the 22-gauge needle was tightened to standardize the degree of stenosis. A video of the surgical procedure is included as Video 1. Control rats underwent the same surgical procedure, without application of the pulmonary artery banding. Clinical signs (eg, dyspnea, weight loss, ruffled fur), were monitored in all animals. One PL rat showed severe unexpected cachexia immediately postsurgery and was therefore excluded from the study. Rats were humanely killed at 4 weeks (control: $\mathrm{n}=5$; PL: $\mathrm{n}=7$ ) or 8 weeks (control: $\mathrm{n}=8$; PL: $\mathrm{n}=10$ ) after surgery (Figure 1, A). Four rats were humanely killed between 3 and 8 weeks after application of PL because of clinical RV failure (humane end point) and could not be analyzed.

\section{NRG1 Administration}

Figure 2, A, shows the schematic outline of the experiment. Wistar rat pups underwent weaning and randomization as described previously (PL: $n=68$; control: $n=29$ ). Subsequently, both groups were randomized into treatment (NRG1) and placebo (vehicle). Ten pups died because of the PL surgery and were therefore excluded (total surgery mortality PL rats: $12.9 \%$ ). rhNRG1 (Peprotech; $60 \mu \mathrm{g} / \mathrm{kg} / \mathrm{d}$, dissolved 


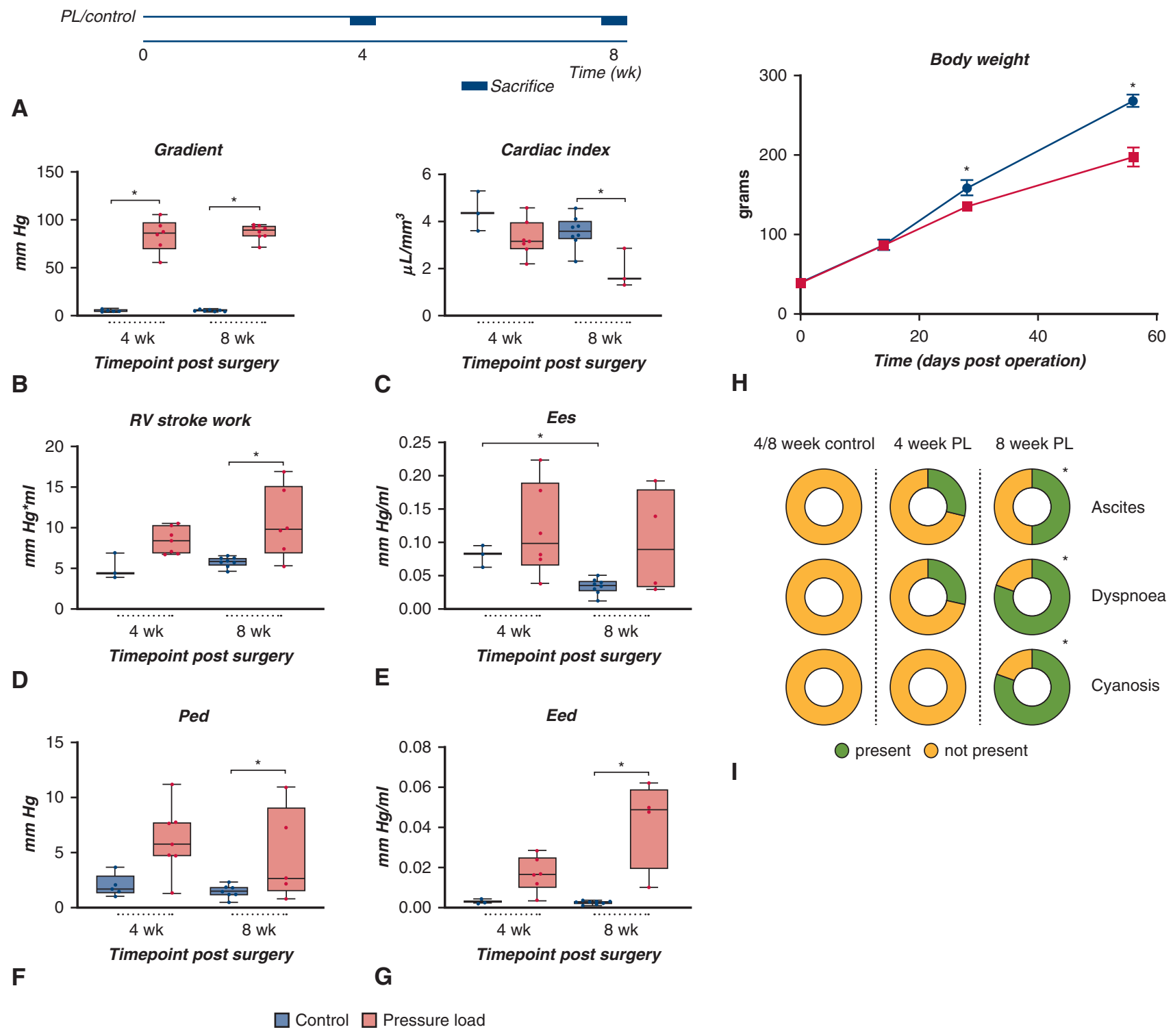

FIGURE 1. A, Schematic overview of experimental design. Surgery in both groups consisted of pulmonary artery banding (PL) or sham surgery (control). Rats were humanely killed 4 or 8 weeks after surgery. B, PL gradient was increased in all groups compared with control. $P$ values: $4 / 8$ week control versus PL: $<.001 /<.001$; control/PL 4 weeks versus 8 weeks: 1.000/.918. C, Decreased cardiac index in PL. $P$ values: 4/8 week control versus PL: .468/.032; control/PL 4 weeks versus 8 weeks: .776/.096. D, Right ventricle $(R V)$-PL increased RV stroke work after 8 weeks, measured according to echocardiographic stroke volume and pressure measurements with an RV pressure-volume catheter. $P$ values: 4/8 week control versus PL: .268/.045; control/PL 4 at weeks versus 8 weeks: $1.000 / 1.000$. E, Large variation in RV contractility measured with end-systolic elastance in the PL rats. $P$ values: $4 / 8$ weeks control versus PL: 1.000/.340; control/PL at 4 weeks versus 8 weeks: .048/.905. F, G, Indices of diastolic dysfunction progressively worsen in PL rats. $P$ values: $4 / 8$ weeks control versus PL: .120/.004; control/PL at 4 weeks versus 8 weeks: $1.000 / .628$. $P$ values: $4 / 8$ weeks control versus PL: . 192/.048; control/PL 4 weeks versus 8 weeks: .921/.096. H, RV-PL impaired body weight growth, starting at 4 weeks. $P$ values: $2 / 4 / 8$ weeks control versus PL: $1.000 / .003 /<.001$. I, Progressive increase in signs of clinical RV failure over time in PL rats. Control rats did not show signs of RV failure. The circles indicate the total number of rats with PL surgery. Ascites: $P=.045$, dyspnoea: $P=.038$, pleural effusion: $P=.001$. In some groups, numbers are lower than mentioned in methods because of failed analyses. Red bars represent PL rats, blue bars represent control rats. The lower and upper borders of the box are the lower and upper quartiles. The middle horizontal line represents the median. The whiskers are the minimum and maximum values. Dots in the box-and-whisker dot plots represent individual measurements. Statistical analysis for B-H: Kruskal-Wallis with correction for multiple testing; I: $\chi^{2}$ test. PL, Pressure load; Ees, end-systolic elastance; $P e d$, end diastolic pressure; $E e d$, end-diastolic elastance. $* P<.05$. 


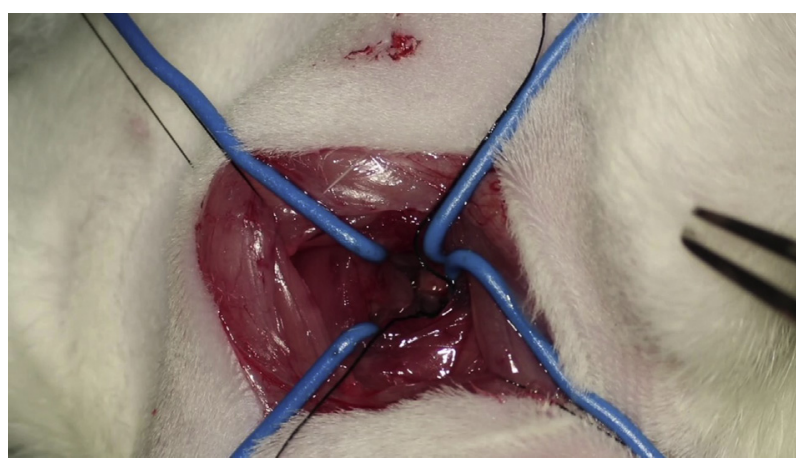

VIDEO 1. Presentation of the surgical procedure of pulmonary artery banding in rats. Because of ethical restrictions, the experimental animal in this video is an adult rat. The procedure is identical in 3-week-old rat pups. The size of the needle in the video is $19 \mathrm{G}$ (22G in rat pups). Video available at: https://www.jtcvs.org/article/S0022-5223(21)01520-8/ fulltext.

in $0.1 \%$ Bovine Serum Albumin/Milli-Q water) or vehicle $(0.1 \%$ Bovine Serum Albumin/Milli-W water) was administered daily to rats through intraperitoneal injection from time $(t)=3$ until $t=14$ days. Animals were randomized into different groups depending on planned time of humane killing (planned humane killing at time point 2 weeks [vehicle: control, $\mathrm{n}=6$; $\mathrm{PL}, \mathrm{n}=9$; NRG1: control, $\mathrm{n}=5$; $\mathrm{PL}$, $\mathrm{n}=10$ ], 4 weeks [vehicle: control, $\mathrm{n}=6 ; \mathrm{PL}, \mathrm{n}=7$; NRG1: PL, $\mathrm{n}=10$ ], or 8 weeks after surgery [vehicle: control $\mathrm{n}=6, \mathrm{PL} \mathrm{n}=11$. NRG1: control $n=6$, PL $n=11]$ ). Clinical signs (eg, dyspnea, weight loss, ruffled fur), were monitored in all animals.

\section{Echocardiography and Pressure-Volume Measurements}

We assessed PL according to maximum flow velocity over the pulmonary artery banding with echocardiography in all animals at 2,4 , and 8 weeks after surgery and assessed cardiac function before humane killing (Vivid Dimension 7; 10S-transducer [GE Healthcare]) as described previously. ${ }^{5}$ Rats were anesthetized with isoflurane (induction $5 \% ; 2 \%-3 \%$ maintenance) and warmed at $37^{\circ} \mathrm{C}$.

Maximum flow velocity over the pulmonary artery banding, left ventricular cardiac output, left ventricular stroke volume, end-systolic eccentricity index, and tricuspid annular plane systolic excursion were derived using the parasternal long axis, short axis (at aortic and midpapillary level), apical 4chamber, and 5-chamber view. Cardiac output was calculated as follows: $(\text { aortic diameter })^{2} \times 3.14 \times$ velocity-time integral $\times$ heart rate. Beat-tobeat variation was accounted for by averaging measurements from 6 to 12 consecutive beats. Cardiac index was calculated using tibia length as described previously. ${ }^{14}$

Before humane killing, open chest pressure-volume measurements were performed in anesthetized and intubated rats using a pressure-admittance catheter (1.9-French, 6-mm spacing; Transonic), as described previously. ${ }^{5}$ After bilateral thoracotomy and pericardiotomy, the catheter was introduced in the apex of the right ventricle toward the outflow tract. The pressure signal was recorded in Chartlab 5 with an ADVantage PV system (ADV500) processor (Transonic).

Analysis of the obtained signals was performed using custom-made software (Circlab 2012, P. Steendijk) to derive RV end-diastolic pressure, $\mathrm{RV}$ peak pressure, and RV maximum and minimum delta pressure and delta time. Stroke volume measured using echocardiography was used to calibrate the conductance-derived stroke volume. Steady-state pressure-volume loops were used for the calculation of all parameters. End-systolic and end-diastolic elastance were determined using the single-beat method as described previously. ${ }^{5}$

\section{Organ Weights and Histological Analyses}

After catheterization, the rats were euthanized by blood withdrawal. After flushing the heart with $\mathrm{NaCL} 0.9 \%$, the heart and organs were removed. The right ventricle, ventricular septum, left ventricle, and both atria were dissected and weighed. After fixation of the right ventricle (formalin), cardiomyocyte cross-sectional area (wheat germ agglutinin), fibrosis (Masson Trichrome), and capillary density (Lectin) were assessed in the RV free wall as described previously. ${ }^{5}$

\section{Immunohistochemistry}

To analyze CCA, 4- $\mu$ m paraffin-embedded RV free wall sections were stained using the following primary antibodies: Ki-67 (1:200, BD Pharmingen) and histone-3-phosphate ([H3P] 1:100; Sigma). To analyze cardiomyocyte-specific CCA, pericentriolar material 1 ([PCM-1] 1:400, Atlas Antibodies) in combination with Ki-76 was used. For PCM-1 counting, Alexa 647 and Alexa 555 were used (1:250) as secondary antibodies. Pictures were taken with a Leica DM600 microscope, 10× magnification. Pictures of H3P staining were taken with a Hamamatsu scanner, $20 \times$ magnification. Nuclei were counted with ImageJ. Per sample, \pm 1500 nuclei were counted.

\section{Gene Expression}

Changes in gene expression in the RV myocardium were analyzed using whole transcriptome expression profiling (RNAseq) as described in Appendix E1. Raw read files are submitted to the National Center for Biotechnology Information Gene Expression Omnibus online database (Gene Expression Omnibus submission, GSE143745; National Center for Biotechnology Information tracking system 20583856).

Quantitative real-time polymerase chain reaction was performed as previously described. ${ }^{5}$ The primer sequences used are included in Table E1. Expression data were corrected for the expression of the reference gene, glyceraldehyde 3-phosphate dehydrogenase.

\section{Statistical Analyses}

The Kruskal-Wallis test was used to analyze differences between more than 2 independent groups. In case of significance, Mann-Whitney $U$, corrected for multiple testing, was used with Bonferroni post hoc analysis to evaluate the specific differences between groups (SPSS statistics, version $23,2015)$. The $\chi^{2}$ test was used for comparisons of categorical variables between independent groups. The assumption of expected cell counts was validated of observing at least 5 counts per cell. NRG1 administration did not affect any of the parameters assessed in rats without PL (tested with Mann-Whitney $U$ ). Therefore, for statistical analyses, data for control animals with and without NRG1 treatment were pooled at each time point and presented as 1 group. Mortality was defined as unplanned humane killing when the humane end point was reached or death.

\section{RESULTS \\ Characterization}

The PL of the right ventricle in the banded pups increased progressively over time as shown by increased Doppler maximal flow velocity over the pulmonary trunk compared with control rats (Figure 1, $B$ ). PL rats had reduced cardiac index at 8 weeks after banding compared with control rats (Figure 1,C). PL led to increased RV stroke work at 8 weeks (Figure $1, D$ ). RV end-systolic elastance showed a large variation and as a group. No increase in end-systolic 
elastance was shown at 4 or 8 weeks of PL (Figure 1,E). PL worsened RV end-diastolic elastance and progressively increased RV diastolic pressure (Figure 1, $F$ and $G$ ). PL decreased tricuspid annular plane systolic excursion and increased eccentricity index, both echocardiographic parameters of RV dysfunction (Table 1).

Four weeks after banding, weight gain was lower compared with control rats (Figure 1, H). At 8 weeks, PL rats showed clinical signs of RV dysfunction (ie, dyspnoea, ruffled fur, ascites, and pleural effusion; Figure 1,I).

PL induced RV hypertrophy, measured as RV weight and cardiomyocyte cross-sectional area (Table 1, Figure 3, A). PL increased RV fibrosis at 8 weeks (Figure 3, B). PL increased capillary:myocyte ratio at 4 and 8 weeks (Table 1).

Immunohistochemistry revealed decreasing cell cycle activity from 4 to 8 weeks in RV tissue of control and PL rats, on the basis of the assessment of Ki-67 staining, which is expressed during all phases of the cell cycle, except the G0 phase (resting phase). ${ }^{15}$ PL tended to increase the number of cells positive for Ki-67 staining (Table 1). To assess whether this cell cycle activity was also present in cardiomyocytes, we used double-staining with PCM-1, a cardiomyocyte-specific nuclear marker. ${ }^{16} \mathrm{PL}$ increased the amount of $\mathrm{Ki}-67 / \mathrm{PCM}-1$ double-positive cells in the right ventricle of PL rats compared with control rats after 4 weeks. This increase declined significantly over time (Figure 3,C). To further assess cell cycle activity in cardiac tissue we stained for H3P (Table 1), but no important differences were observed for PL and control rats. Markers of cardiac stress, natriuretic pro-peptide $\mathrm{A} / \mathrm{B}$, and myosin heavy chain isoform switch, increased at 4 and 8 weeks of PL. Similarly, heme oxygenase 1, a marker of oxidative stress and metallopeptidase inhibitor 1, a metallopeptidase inhibitor and pro-fibrotic marker, were increased (Figure 3,D).

To dissect pathways associated with maturation and adaptation to PL, we performed RNA sequencing analyses using RV tissue 4 and 8 weeks after surgery. Principle component analysis showed differential clustering around control versus PL (Figure E1, A). Within the groups PL and control, principal component analysis showed clustering around time after surgery (4 vs 8 weeks). At a cutoff of $>1 \log 2$-fold change, 5 clusters of genes were differentially expressed. These clusters involved a wide range of processes (Figure 4, A). Within the control and PL groups, we found maturational differences. Gene ontology analysis indicated that genes with higher expression in control rats at 4 weeks versus 8 weeks were mainly related to cell cycle, whereas genes with higher expression in control rats after 8 weeks versus 4 weeks were mainly related to metabolism (mitochondrion, fatty acid metabolism, peroxisome, tricarboxylic acid cycle; Figure 4, $B$ ).

Within the group of PL rats, we identified maturational differences between 4 and 8 weeks (ie, changes in transcriptional profiles related to change from "fetal program" toward "mature cardiomyocytes.") To assess the effect of PL on this development from fetal to adult programs, we specifically selected genes that were differentially expressed in PL rats versus control rats at 4 weeks and 8 weeks (Figure E1, $B$ ). The genes with increased expression after 4 weeks of PL are involved in cell division in mitosis, similar to the differences at 4 and 8 weeks in control rats. The genes that were increased only at 8 weeks of PL are mostly involved in immunity.

PL also induced a time-independent up-regulation of many known candidates affected in hypertrophy. A comparison of PL rats with control rats revealed up-regulated genes belonging to the processes of, among others, stress response and cell division, whereas down-regulated genes were those belonging to processes of metabolism. These changes were largely similar after 4 and 8 weeks of PL. Several changes in gene expression were confirmed with quantitative polymerase chain reaction (natriuretic pro-peptide $\mathrm{B}$, myosin heavy chain $7[M y h 7]$, myosin heavy chain $6[M y h 6]$; Figure $4, C$ ).

\section{Treatment With NRG1}

The results of the study show no differences for NRG1 control and vehicle control groups at time points 2 and 8 weeks. In light of reduction of experimental animals, we therefore made the assumption that no differences would be found among the control groups at time point 4 weeks.

The applied pulmonary banding induced equal RV PL in vehicle- and NRG1-treated rats, measured according to increased Doppler maximal flow velocity (Figures 2, B, and E2). NRG1 treatment after surgery restored cardiac index in PL rats, to values comparable with rats without PL (Figure 2, C). NRG1 treatment also increased RV stroke work in PL rats, but did not affect RV end-systolic elastance (Figure 2, $D$ and $E$ ). We believe NRG1 treatment tends to improve diastolic function because end-diastolic elastance was lower at 2 weeks, although the statististical analysis used did not support this finding (Figure 2, G). This effect weaned at 4 and 8 weeks. NRG1 did not affect other echocardiographic indices of RV function (Tables 2-4).

Between 4 and 8 weeks, 13 rats with PL developed severe clinical RV failure or death and were humanely killed (vehicle: 7 rats; NRG1: 6 rats). Treatment with NRG1 in PL rats postponed the age of death (time after surgery) and/or development of severe RV failure (Figure 2, $H$ ). NRG1 treatment did not affect weight gain in the PL rats.

NRG1 treatment decreased the progression of fibrosis development in RV PL rats at 4 and 8 weeks. Other markers of RV remodeling, hypertrophy and capillary:myocyte ratio, were not affected by NRG1 treatment (Figure 5, A and $B$, Tables 2-4).

NRG1 treatment increased cardiomyocyte-specific Ki67. The increase in fraction of Ki-67 cardiomyocytes was 


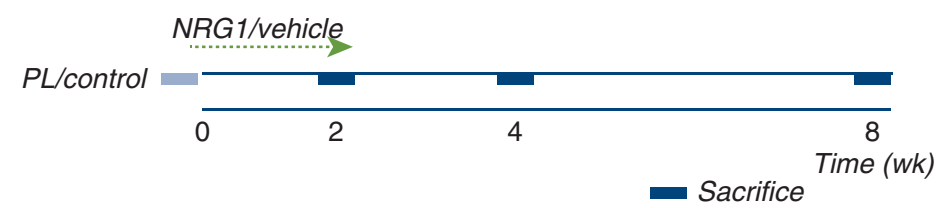

A

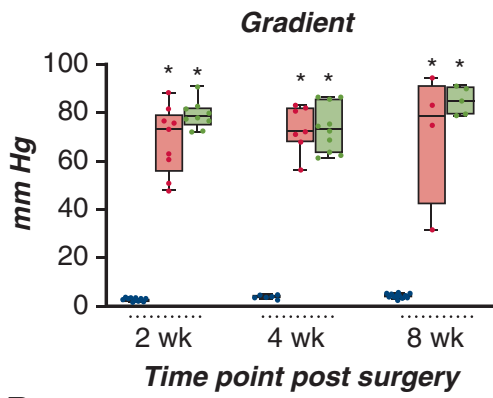

B

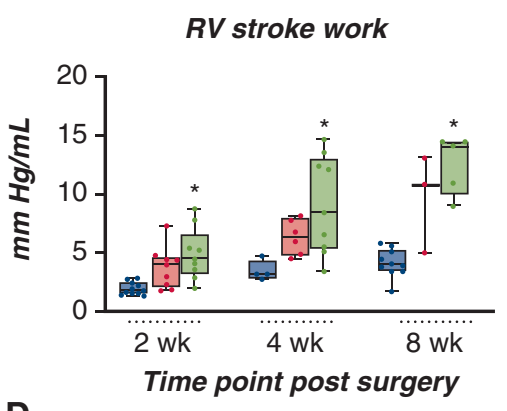

D

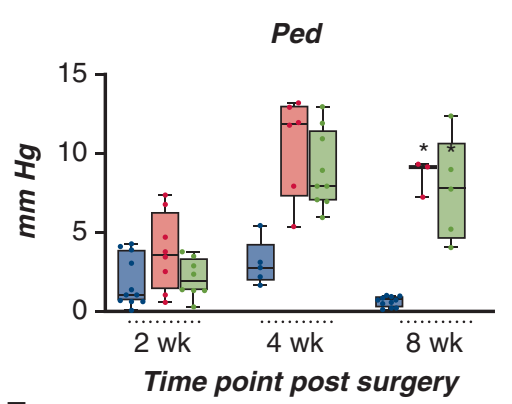

$\mathbf{F}$

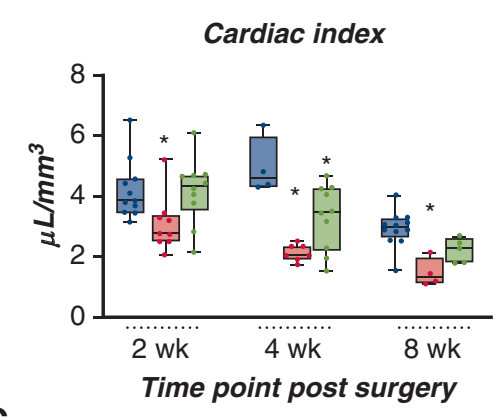

Ees

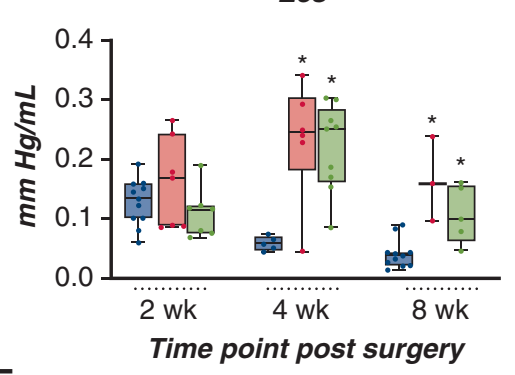

E

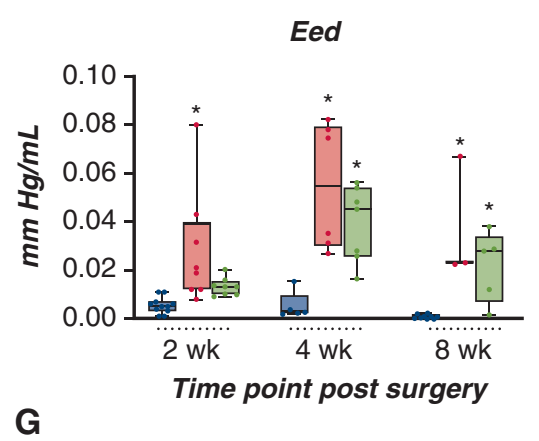

Mean age at time of development severe RV failure

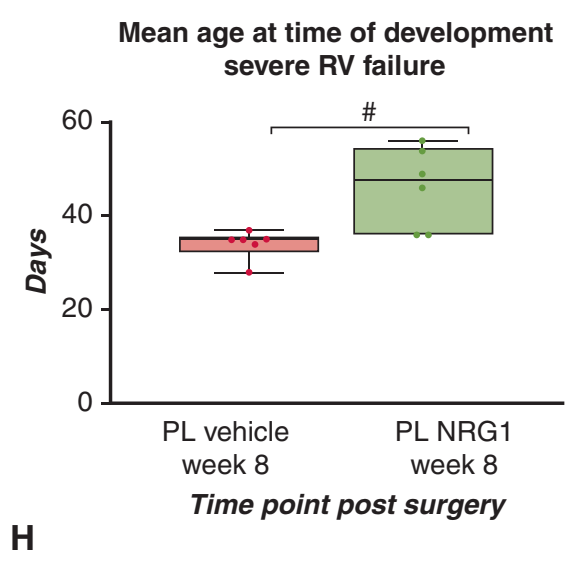

H 
TABLE 1. Characteristics of the experimental rat groups

\begin{tabular}{|c|c|c|c|c|c|c|}
\hline & \multicolumn{3}{|c|}{4 Weeks } & \multicolumn{3}{|c|}{8 Weeks } \\
\hline & Control & PL & $\overline{\mathbf{P} 1}$ & Control & PL & P 2/3/4 \\
\hline Number of rats & 5 & 7 & & 8 & 9 & \\
\hline \multicolumn{7}{|l|}{ Weight, $g$} \\
\hline LV + IVS & $0.35(0.04)$ & $0.55(0.05)$ & .418 & $0.58(0.08)$ & $0.59(0.15)$ & $1.000 / 1.000 / .608$ \\
\hline $\mathrm{RV} /(\mathrm{LV}+\mathrm{IVS})$ & $0.35(0.04)$ & $0.55(0.06)^{*}$ & .012 & $0.30(0.06)$ & $0.68(0.21)^{*}$ & $<.001 / 1.000 / 1.000$ \\
\hline RA & $0.04(0.04)$ & $0.16(0.5)^{*}$ & .012 & $0.11(0.14)$ & $0.17(0.12)^{*}$ & $.260 / .508 / 1.000$ \\
\hline \multicolumn{7}{|l|}{ Echocardiographic parameters } \\
\hline Eccentricity index diastolic & $0.9(0.4)$ & $1.6(0.3)$ & .068 & $0.9(0.3)$ & $1.6(0.3)$ & $.120 / .497 / 1.000$ \\
\hline TAPSE, $\mathrm{mm}$ & $2.9(0.7)$ & $2.2(0.5)$ & .468 & $3.0(0.6)$ & $2.9(1.2)$ & $.464 / .776 / 1.000$ \\
\hline Cardiac output, $\mathrm{mL} / \mathrm{min}$ & $108.8(5,6)$ & $66.0(13,8)$ & .068 & $110.6(23.2)$ & $68.2(29.4)^{*}$ & $<.001 / .096 / .805$ \\
\hline \multicolumn{7}{|l|}{ Histology } \\
\hline RV Ki-67 positive nuclei, \% & $5.78(1,54)$ & $8.33(1,56)$ & .09 & $2.54(0.45) \dagger$ & $4.40(1.98) \dagger$ & $.004 / .012 / .036$ \\
\hline $\begin{array}{l}\text { RV histone-3-phosphate } \\
\text { nuclei, \% }\end{array}$ & $0.14(0.09)$ & $0.20(0.05)$ & .639 & $0.19(0.06)$ & $0.22(0.48)$ & $1.000 / .684 / 1.000$ \\
\hline Capillary-myocyte ratio & $0.81(0.20)$ & $0.95(0.18)^{*}$ & .040 & $0.69(0.20)$ & $1.16(0.30)^{*}$ & $<.001 / 1.000 / .204$ \\
\hline
\end{tabular}

Data are presented as mean (SEM), except where otherwise noted. $P L$, Pressure load; $P 1, P$ value control versus pressure load at 4 weeks; $P 2$, value control versus pressure load at 8 weeks; $P 3, P$ value control at 4 weeks versus control at 8 weeks; $P 4, P$ value pressure load at 4 weeks versus pressure load at 8 weeks; $L V$, left ventricle; $I V S$, interventricular septum; $R V$, right ventricle; $R A$, right atrium; TAPSE, tricuspid annular plane systolic excursion. $* P<.05$ control versus PL at same time point. $\dagger P<.05$ same intervention at different time points.

most prominent after 2 weeks but still present after 4 and 8 weeks, albeit at a lower size (Figure 5, C). Quantitative polymerase chain reaction did not show differences between vehicle and NRG1 treatment in fetal gene switch (Myh7:Myh6 ratio; Tables 2-4), genes associated with cardiomyocyte proliferation (runt-related transcription factor 1, Disabled adaptor protein 2) or metabolism (medium-chain acyl-CoA dehydrogenase, glucose transporter 1). Metallopeptidase inhibitor 1, involved in fibrosis, tended to be lower in the NRG1 treatment group $(P=.051$; Figure 5, D).

\section{DISCUSSION}

In this study, we used a rat pup model for chronic, increased RV PL from immaturity into adulthood to mimic cardiac adaptation in patients with CHD. RV PL in rat pups induced cell cycle activity and delayed transcriptional maturation. After 4 weeks of initial adaptation, progressive failure to thrive, RV dysfunction, and RV fibrosis developed. Increased cell cycle activity via exogenous administration of the growth factor, NRG1, improved cardiac function, and delayed the onset of RV fibrosis and failure. This proof-of-concept study suggests that stimulation of cell cycle activity during growth enhances RV adaptation to PL and might provide a new approach to preserve RV function in CHD.

In this study we modeled adaptation to PL during growth and development from "early childhood" into "adulthood." This new approach has been developed in rats and pigs. ${ }^{17,18}$ The use of such models is especially relevant for CHD, where the heart has to adapt to PL conditions during physical maturation. Throughout this study, the body mass increased from $30 \mathrm{~g}$ to $300 \mathrm{~g}$, a growth rate comparable with humans during childhood. ${ }^{13}$ Our study extends recently described observations from PL in newborn rat pups ${ }^{18}$ in which a gradient of $8 \mathrm{~mm} \mathrm{Hg}$ was applied, compared with $80 \mathrm{~mm} \mathrm{Hg}$ in the present study. In that study, the initial adaptation to the mild PL applied on postnatal day 1 was RV hypertrophy without fibrosis. The age and weight of the newborn rats precluded further cardiac performance measurements as performed in this study. Other rat models, in which PL was applied at an older age (eg, body weight of 80-100 g) yielded different age-dependent responses. ${ }^{17}$ Chronic PL applied at an older age eventually induces RV dysfunction, fibrosis, and remodeling, which has been previously characterized. ${ }^{5}$ These signals have also been identified in children with RV PL (eg, tetralogy of Fallot) in tissues obtained at primary surgery. ${ }^{19}$ These experimental models of chronic RV PL during growth and development

rats, green bars represent PL NRG1 rats, blue bars represent control rats. In some groups, numbers are lower than mentioned in methods because of failed analyses. The lower and upper borders of the box are the lower and upper quartiles. The middle horizontal line represents the median. The whiskers are the minimum and maximum values. Dots in the box-and-whisker dot plots represent individual measurements. Statistical analysis for B-G: Kruskal-Wallis with correction for multiple testing, and H: Mann-Whitney U. PL, Pressure load; Ees, end-systolic elastance; Ped, end diastolic pressure; Eed, end-diastolic elastance. $* P<.05$ compared with control. 

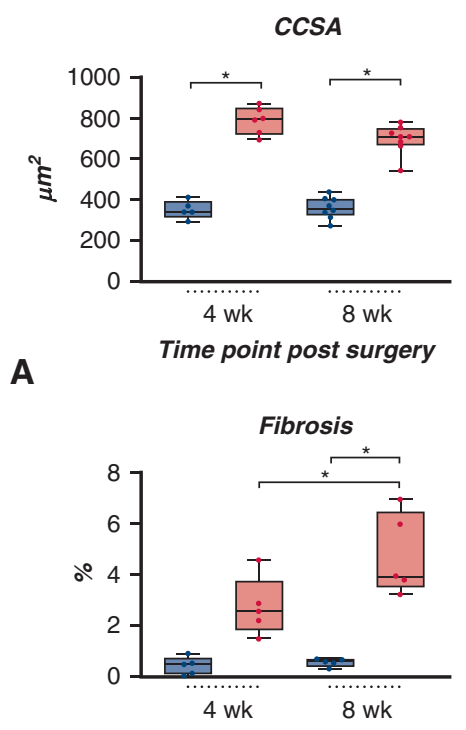

B

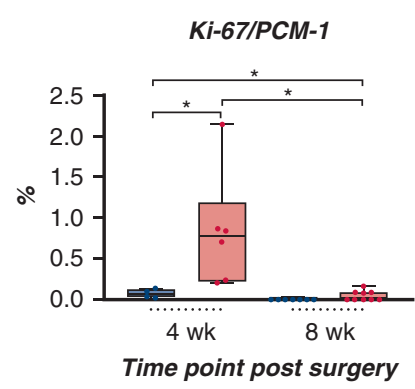

C
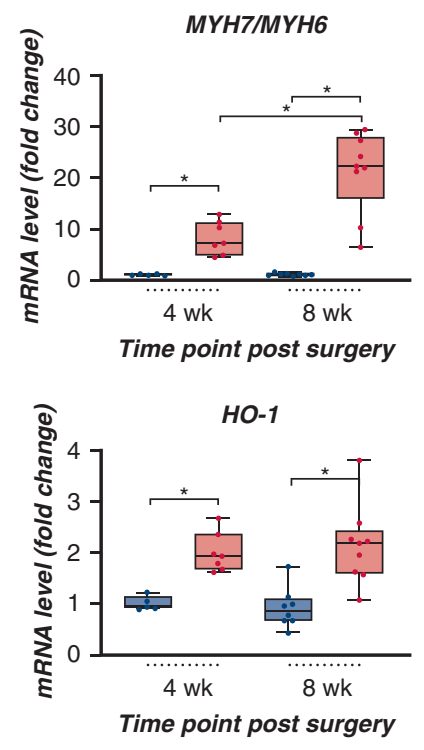

D
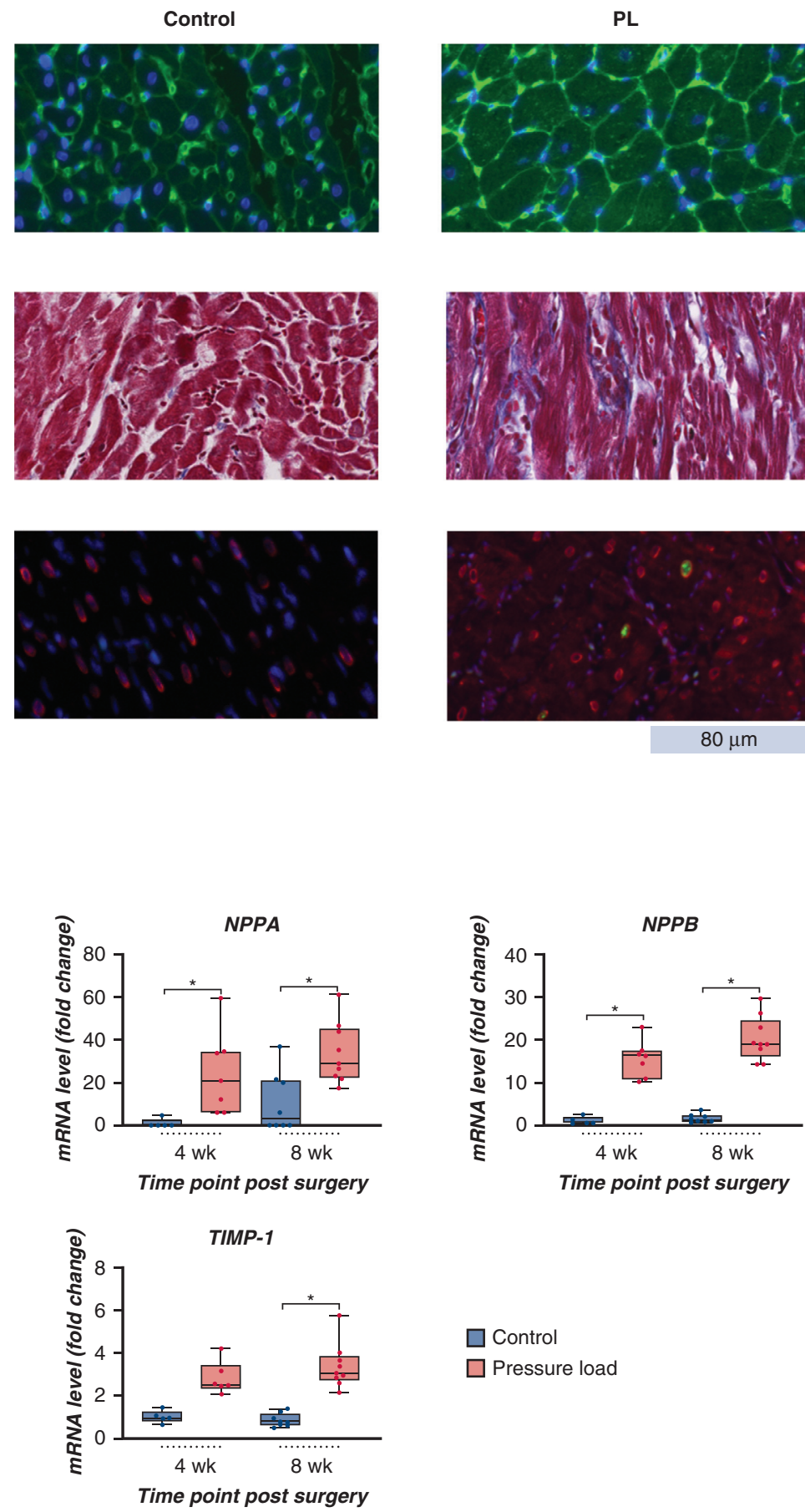
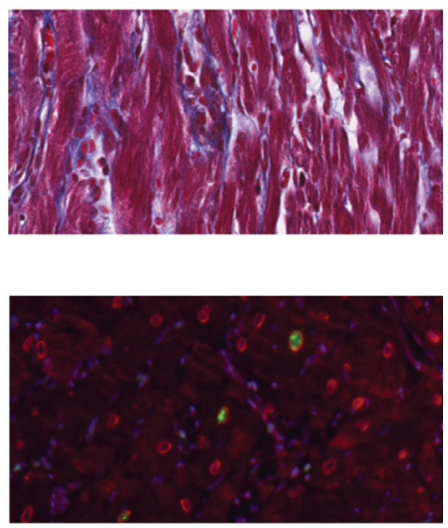

$80 \mu \mathrm{m}$

PL
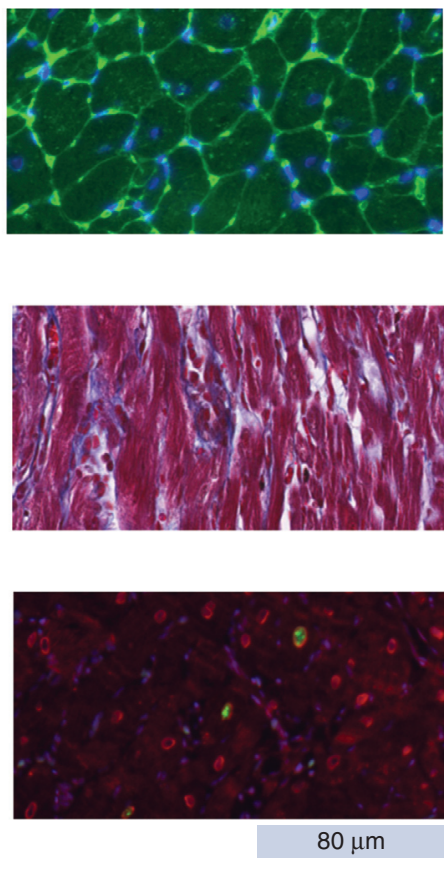

Time point post surgery

FIGURE 3. Histology and gene expression. A, Pulmonary artery banding (PL) increased right ventricular (RV) hypertrophy expressed by cardiomyocyte cross-sectional area (CCSA; first row are representative images of wheat germ agglutinin-stained RVs 4 weeks after surgery). $P$ values: $4 / 8$ weeks control versus PL: .016/<.001; control/PL at 4 weeks versus 8 weeks: 1.000/.140. B, fibrosis increased in PL rats at 8 weeks (second row images are representative images of Masson-Trichrome-stained RVs 8 weeks after surgery). $P$ values: $4 / 8$ weeks control versus PL: $.468 /<.001$; control/PL at 4 weeks versus 8 weeks: $.096 / .008$. C, RV-PL increased cardiomyocyte specific cell-cycle activity and decreases over time in control and PL rats. Representative slides of DAPI (blue)/Ki-67 (green)/pericentriolar material 1 (PCM-1, red) staining in the RV at 4 weeks of control and PL right ventricles. Colors were enhanced for 


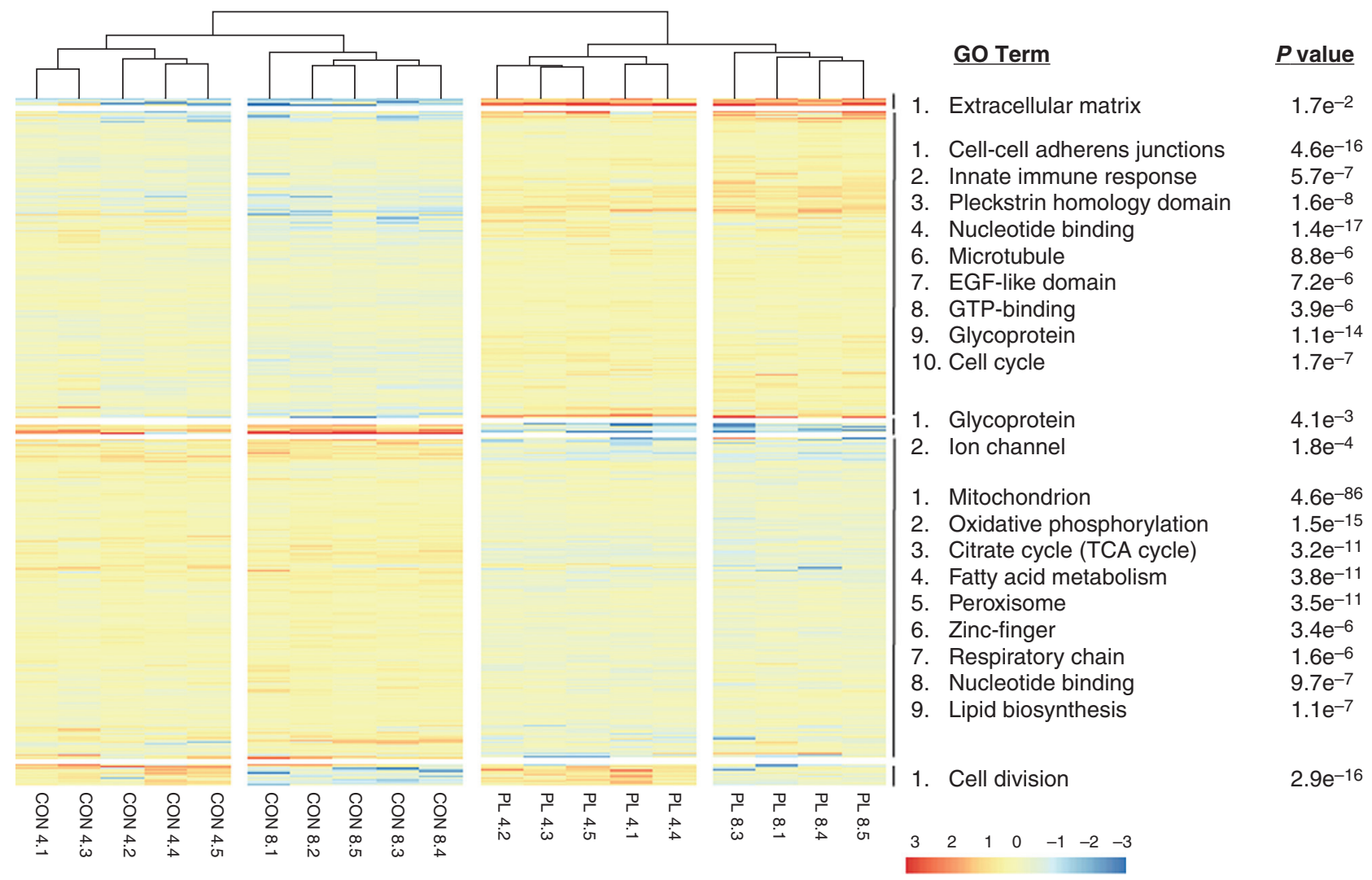

A

FIGURE 4. RNA sequencing identifies delayed silencing of fetal pathways. A, Functional annotated heat map of pulmonary artery banding (PL) versus control $(C O N)$ at 2 time points (4 weeks and 8 weeks). Gene ontology analysis was performed on each cluster. B, Volcano plot showing cardiac transcripts differentially expressed between 4 and 8 weeks in control (top left), PL (bottom left) and between control and PL at 4 weeks (top right) and 8 weeks (bottom right). Colored dots indicate significantly differentially expressed genes ( $P$ adjusted for multiple testing; false discovery rate $<.05)$. $P L$, Pressure load; $G O$, gene ontology; EGF, epidermal growth factor; GTP, guanosine 5'-triphosphate; TCA, tricarboxylic acid cycle.

show similarities to children with CHD, and might create a window of opportunity to search for potential therapies during childhood. In accordance with patients with severe CHD, this model of RV PL induced failure to thrive and signs of clinical RV failure after a relative incident-free youth. ${ }^{20}$ This model underscores that diastolic dysfunction is a leading cause for the development of clinical right heart failure. ${ }^{17,21}$ Known markers of $\mathrm{RV}$ failure in adult PL models (eg, natriuretic pro-peptide $\mathrm{A} / \mathrm{B}$ levels and
Myh7:Myh6 ratio) also increase in young PL rats. ${ }^{4}$ In patients with tetralogy of Fallot, increased stiffness and PL are accompanied by increased signaling involved in muscle tissue development, ventricle contractility, and calcium signaling. ${ }^{19}$ These signals also arose in the expression profiles of RV PL tissue.

A major finding in the present study was the delay in transcriptional maturation and increase in cell cycle activity observed during growth and development in the pups with

analysis and presentation. $P$ values: 4/8 weeks control versus PL: .040/.484; control/PL at 4 weeks versus 8 weeks: .024/.004. D, mRNA expression of genes from left to right related to fetal gene switch, stress markers, and fibrosis (control 4 weeks $=1) . \beta / \alpha$-Myosin heavy chain switch $(M Y H 7 / 6) P$ values: $4 /$ 8 weeks control versus PL: .012/<.001; control/PL at 4 weeks versus 8 weeks: $1.000 / .032$. Natriuretic pro-peptides type A (NPPA) $P$ values: $4 / 8$ weeks control versus PL: .012/.024; control/PL at 4 weeks versus 8 weeks: 1.000/.840. Natriuretic pro-peptides type B (NPPB) $P$ values: $4 / 8$ weeks control versus PL: .012/<.001; control/PL at 4 weeks versus 8 weeks: .888/.364. Heme oxygenase (HO- 1$) P$ values: 4/8 weeks control versus PL: .012/.004; control/PL at 4 weeks versus 8 weeks: 1.000/1.000. Metallopeptidase inhibitor 1 (TIMP-1) $P$ values: 4/8 weeks control versus PL: .192/<.001; control/PL at 4 weeks versus 8 weeks: 1.000/.465. Red bars represent PL rats, blue bars represent control rats. The circles indicate the total number of rats with PL surgery. In some groups, numbers are lower than mentioned in methods because of failed analyses. The lower and upper borders of the box are the lower and upper quartiles. The middle horizontal line represents the median. The whiskers are the minimum and maximum values. Dots in the box-and-whisker dot plots represent individual measurements. Statistical analysis: Kruskal-Wallis with correction for multiple testing. *P<.05. 

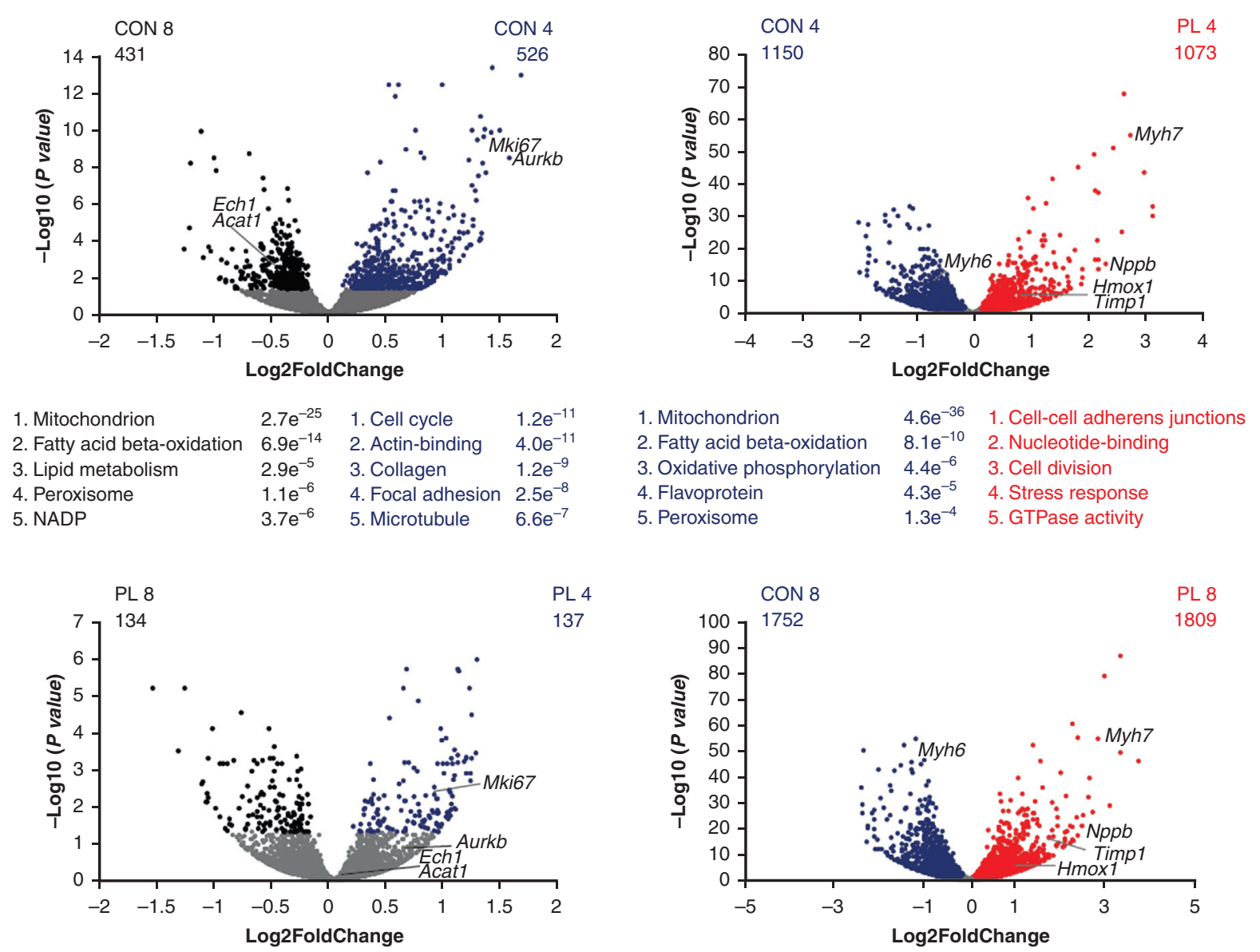

$\begin{array}{llll}\text { 1. Hemoglobin complex } & 5.4 \mathrm{e}^{-6} & \text { 1. Cell cycle } & 8.9 \mathrm{e}^{-9} \\ \text { 2. Immunity } & 6.3 \mathrm{e}^{-4} & \text { 2. Methylation } & 2.2 \mathrm{e}^{-4} \\ & & \text { 3. Chromosome } & 1.9 \mathrm{e}^{-13} \\ & & \text { 4. Mitosis } & 8.0 \mathrm{e}^{-8} \\ & & \text { 5. Cell cycle } & 2.5 \mathrm{e}^{-8}\end{array}$

PL 4

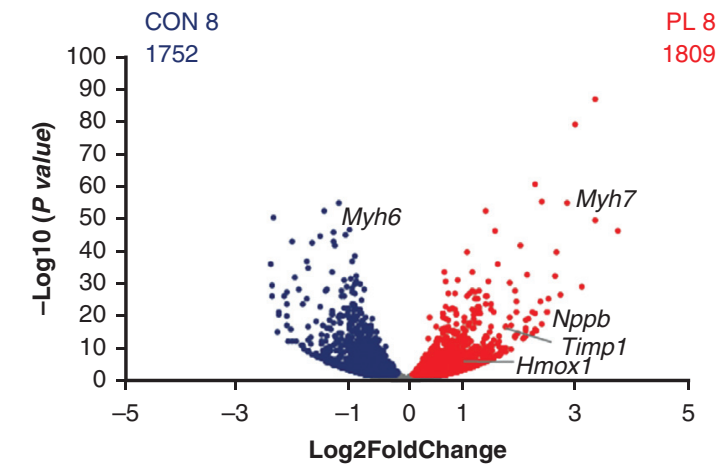

137

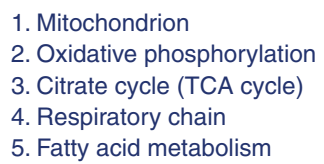

$3.6 \mathrm{e}^{-97} \quad$ 1. Cell-cell adherens junctions

$1.9 \mathrm{e}^{-20} \quad$ 2. Innate immune response

$1.0 \mathrm{e}^{-12} \quad$ 3. Glycoprotein

$6.6 \mathrm{e}^{-8} \quad$ 4. EGF-like domain

$1.2 \mathrm{e}^{-10} \quad$ 5. Pleckstrin homology-like domain $1.8 \mathrm{e}^{-8}$

FIGURE 4. Continued.

PL. Since the initial description of postnatal cardiomyocyte proliferation, ${ }^{22,23}$ many investigators have studied this phenomenon. ${ }^{23,24}$ In the study applying increased PL to 1-day-old rat pups a similar profile of delay in maturation and activation of proliferation signals was measured. ${ }^{18}$ Several postnatal processes might be involved in the loss of proliferative potential with age, (eg, increase in oxygen saturation). ${ }^{25}$ The functional consequences of these transcriptional changes for RV adaptation are still elusive. We introduced a growth factor, NRG1, to further study the consequences of increased CCA on chronic PL in the postnatal developing heart.

Since the first observation of that NRG1 might enhance CCA in vitro, ${ }^{26}$ few studies have assessed the effects of NRG1 in vivo in acute stress models. ${ }^{27}$ This study extends the observation that in vivo administration of NRG1 in rat pups with chronic PL increases CCA and improves RV cardiac performance. These findings implicate first that the transcriptional changes involved in cell cycle activity observed in rat pups with PL are positive coping mechanisms. Further stimulation of CCA via NRG1 improves cardiac adaptation to PL. Another finding is the delayed onset of cardiac fibrosis in NRG1-treated PL rats. It is known that NRG1 treatment might also induce paracrine effects via cardiac fibroblasts in vivo ${ }^{28}$ and increase phosphorylation of phospholamban. ${ }^{29}$ In vitro, NRG1 decreases RV fibrosis in adult PL rats. ${ }^{30}$ However, this did not result in improvement of cardiac function. It should be noted that RV fibrosis is part of the adaptive as well as maladaptive response to increased RV afterload. Previous studies in adult mice and rats suggested that $\mathrm{RV}$ dysfunction is not directly related to RV fibrosis, yet fibrosis might be interpreted as a sign 
TABLE 2. Values at 2 weeks

\begin{tabular}{|c|c|c|c|c|c|c|c|c|}
\hline & Control & $\mathbf{n}$ & PL veh & $\mathbf{n}$ & P 1 & PL NRG1 & $\mathbf{n}$ & P $2 / 3$ \\
\hline Body weight, $g$ & $82.4(9.5)$ & 28 & $73.3(7.5)$ & 25 & .393 & $70.1(9.5)$ & 31 & $.297 / .780$ \\
\hline Tibia length, mm & $23.6(0.7)$ & 11 & $22.9(1.2)$ & 9 & .456 & $22.9(1.3)$ & 10 & $.519 / .968$ \\
\hline \multicolumn{9}{|l|}{ Organ weights, g } \\
\hline RV & $0.08(0.03)$ & 11 & $0.18 *(0.03)$ & 9 & $<.001$ & $0.15^{*}, \dagger(0.03)$ & 10 & $<.001 / .009$ \\
\hline $\mathrm{LV}+\mathrm{IVS}$ & $0.27(0.03)$ & 11 & $0.29(0.03)$ & 9 & .525 & $0.28(0.06)$ & 10 & $1.000 / 1.000$ \\
\hline $\mathrm{RV} /(\mathrm{LV}+\mathrm{IVS})$ & $0.29(0.03)$ & 11 & $0.64 *(0.12)$ & 9 & $<.001$ & $0.53 *(0.06)$ & 10 & $<.001 / .129$ \\
\hline RA & $0.024(0.013)$ & 11 & $0.075 *(0.033)$ & 9 & $<.001$ & $0.045 *(0.015)$ & 9 & $.012 / .189$ \\
\hline \multicolumn{9}{|l|}{ Echocardiographic parameters } \\
\hline Cardiac output, $\mathrm{mL} / \mathrm{min}$ & $55.3(12.3)$ & 11 & $37.2 *(13.2)$ & 9 & .018 & $49.8(13.0)$ & 10 & $.753 / .066$ \\
\hline TAPSE, $\mathrm{mm}$ & $2.1(0.3)$ & 11 & $1.5^{*}(0.3)$ & 8 & $<.001$ & $1.5^{*}(0.3)$ & 10 & $<.001 / .829$ \\
\hline Eccentricity index diastolic & $1.00(0.07)$ & 11 & $1.64 *(0.05)$ & 6 & $<.001$ & $1.59 *(0.32)$ & 9 & $<.001 / .388$ \\
\hline \multicolumn{9}{|l|}{ Histology } \\
\hline Capillary:myocyte ratio & $0.65(0.10)$ & 11 & $0.87 *(0.14)$ & 8 & .012 & $0.96^{*}(0.16)$ & 10 & $<.001 / .519$ \\
\hline \multicolumn{9}{|l|}{ qPCR } \\
\hline Myh7/6 & $0.99(0.40)$ & 10 & $102.0 *(89.91)$ & 9 & $<.001$ & $44.24 *(38.9)$ & 10 & $<.001 / .129$ \\
\hline
\end{tabular}

Data are presented as mean (SD), except where otherwise noted. $P L$, Pressure load; veh, vehicle; $P 1, P$ value control versus pressure load vehicle; $N R G 1$, neuregulin-1; $P$ 2, $P$ value control versus pressure load neuregulin-1; $P 3, P$ value pressure vehicle versus pressure load neuregulin-1; $R V$, right ventricle; $L V$, left ventricle; $I V S$, interventricular septum; $R A$, right atrium; TAPSE, tricuspid annular plane systolic excursion; $q P C R$, quantitative polymerase chain reaction; $M y h 7 / 6$, myosin heavy chain $7 / 6$. $* P<.05$ control versus $P L$. $\dagger P<.05$ PL vehicle versus PL NRG1.

of maladaption. ${ }^{5,31}$ Potentially, NRG1 can induce side effects on other organs. This has not been the case. Administration of NRG1 during growth and development has not been shown to induce effects on other organs. ${ }^{32}$ These findings underline the therapeutic potential of NRG1 administration in the RV PL during growth and development.

Several approaches have been taken to improve RV adaptation to stress in patients with CHD. ${ }^{33}$ Multiple studies inject either stem cells or cardiospheres into the myocardium or into the coronary artery. None of these studies have shown actual CCA, but some show improvements in ejection fraction and reduction in fibrosis. The approach in the present study yields a noninvasive therapeutic strategy with few side effects and might offer new treatment opportunities, and shows that RV failure might be amenable for therapy focusing on processes involved in cardiac regeneration. NRG1 administration has been tested in adult experimental models and has been tested safe and welltolerated in 2 phase II trials in adult heart failure patients. ${ }^{34}$ Further experiments are needed to study the effects of

TABLE 3. Values at 4 weeks

\begin{tabular}{|c|c|c|c|c|c|c|c|c|}
\hline & Control & $\mathbf{n}$ & PL veh & $\mathbf{n}$ & $P$ & PL NRG1 & $\mathbf{n}$ & $P$ \\
\hline Body weight, $g$ & $149.8(12.4)$ & 16 & $127.4 *(4.9)$ & 16 & .015 & $120.2 *(18.3)$ & 21 & $<.001 / 1.000$ \\
\hline Tibia length, mm & $27.6(0.7)$ & 6 & $26.3 *(0.1)$ & 7 & .024 & $26.1 *(0.3)$ & 10 & $.009 / .945$ \\
\hline \multicolumn{9}{|l|}{ Organ weights, $\mathrm{g}$} \\
\hline RV & $0.10(0.02)$ & 5 & $0.25 *(0.01)$ & 7 & .009 & $0.23 *(0.03)$ & 10 & $.003 / 1.000$ \\
\hline $\mathrm{LV}+\mathrm{IVS}$ & $0.40(0.02)$ & 5 & $0.39(0.02)$ & 7 & 1.000 & $0.48(0.06)$ & 10 & $.618 / .579$ \\
\hline $\mathrm{RV} /(\mathrm{LV}+\mathrm{IVS})$ & $0.10(0.02)$ & 5 & $0.65 *(0.06)$ & 7 & .009 & $0.52 *(0.13)$ & 10 & $.003 / .264$ \\
\hline RA & $0.037(0.017)$ & 5 & $0.147 *(0.010)$ & 7 & .009 & $0.147 *(0.025)$ & 10 & $.003 / 1.000$ \\
\hline \multicolumn{9}{|l|}{ Echocardiographic parameters } \\
\hline Cardiac output, $\mathrm{mL} / \mathrm{min}$ & $107.4(19.0)$ & 4 & $38.7 *(2.0)$ & 7 & .012 & $60.0 *(17.1)$ & 10 & $.006 / .129$ \\
\hline TAPSE $(\mathrm{mm})$ & $3.9(0.8)$ & 4 & $1.4 *(0.1)$ & 6 & .006 & $1.7 *(0.32)$ & 10 & $<.001 / .354$ \\
\hline Eccentricity index diastolic & $0.94(0.09)$ & 5 & $1.83 *(0.13)$ & 7 & .009 & $1.87(0.62)$ & 8 & $.090 / 1.000$ \\
\hline \multicolumn{9}{|l|}{ Histology } \\
\hline Capillary:myocyte ratio & $0.74(0.17)$ & 6 & $1.05 *(0.06)$ & 7 & .024 & $1.06 *(0.19)$ & 10 & $.021 / 1.000$ \\
\hline \multicolumn{9}{|l|}{ qPCR } \\
\hline Myh7/6 & $1.12(0.51)$ & 5 & $154.9 *(63.72)$ & 7 & .009 & $154.7 *(62.9)$ & 10 & $.003 / .690$ \\
\hline
\end{tabular}

Data are presented as mean (SD), except where otherwise noted. $P L$, Pressure load; veh, vehicle; $N R G 1$, neuregulin-1; $R V$, right ventricle; $L V$, left ventricle; $I V S$, interventricular septum; $R A$, right atrium; TAPSE, tricuspid annular plane systolic excursion; $q P C R$, quantitative polymerase chain reaction; $M y h 7 / 6$, myosin heavy chain $7 / 6$. $* P<.05$ control versus PL. 
TABLE 4. Values at 8 weeks

\begin{tabular}{|c|c|c|c|c|c|c|c|c|}
\hline & Control & $\mathbf{n}$ & PL veh & $\mathbf{n}$ & $\boldsymbol{P}$ & PL NRG1 & $\mathbf{n}$ & $\boldsymbol{P}$ \\
\hline Body weight, $g$ & $274.3(19.4)$ & 12 & $206.5^{*}(18,6)$ & 4 & .012 & $214.4 *(20.8)$ & 5 & $.003 / .730$ \\
\hline Tibia length, mm & $34.5(0.3)$ & 11 & $32.3^{*}(1.2)$ & 4 & .012 & $32.4 *(1.1)$ & 5 & $.006 / .730$ \\
\hline \multicolumn{9}{|l|}{ Organ weights, g } \\
\hline RV & $0.16(0.03)$ & 12 & $0.31 *(0.21)$ & 3 & .012 & $0.32 *(0.07)$ & 5 & $<.001 / 1.000$ \\
\hline $\mathrm{LV}+\mathrm{IVS}$ & $0.60(0.03)$ & 12 & $0.57(0.07)$ & 3 & .885 & $0.59(0.11)$ & 5 & $1.000 / .786$ \\
\hline $\mathrm{RV} /(\mathrm{LV}+\mathrm{IVS})$ & $0.27(0.03)$ & 12 & $0.54 *(0.14)$ & 3 & .012 & $0.56 *(0.16)$ & 5 & $<.001 / 1.000$ \\
\hline RA & $0.041(0.014)$ & 12 & $0.225^{*}(0.007)$ & 3 & .012 & $0.241 *(0.022)$ & 5 & $<.001 / 1.000$ \\
\hline \multicolumn{9}{|l|}{ Echocardiographic parameters } \\
\hline Cardiac output, mL/min & $118.1(23.9)$ & 12 & $50.5^{*}(21.2)$ & 4 & .006 & $75.1 *(13,9)$ & 5 & $.012 / .333$ \\
\hline TAPSE, $\mathrm{mm}$ & $3.1(0.3)$ & 12 & $2.55(1.2)$ & 4 & .599 & $2.0^{*}(0.45)$ & 5 & $<.001 / .730$ \\
\hline Eccentricity index diastolic & $1.00 *(0.03)$ & 11 & $1.46^{*}(0.21)$ & 3 & .025 & $1.21 *(0.45)$ & 5 & $<.001 / .571$ \\
\hline \multicolumn{9}{|l|}{ Histology } \\
\hline Capillary:myocyte ratio & $0.99(0.14)$ & 12 & $1.53 *(0.20)$ & 6 & .003 & $1.34 *(0.04)$ & 4 & $.003 / .171$ \\
\hline \multicolumn{9}{|l|}{ qPCR } \\
\hline Myh7/6 & $0.97(0.27)$ & 11 & $17.67(11.2)$ & 3 & .078 & $19.97(2.27)$ & 3 & $.078 / 1.000$ \\
\hline
\end{tabular}

Data are presented as mean (SD), except where otherwise noted. $P L$, Pressure load; veh, vehicle; $N R G 1$, neuregulin-1; $R V$, right ventricle; $L V$, left ventricle; $I V S$, interventricular septum; $R A$, right atrium; TAPSE, tricuspid annular plane systolic excursion; $q P C R$, quantitative polymerase chain reaction; $M y h 7 / 6$, myosin heavy chain $7 / 6$. $* P<.05$ control versus PL.

repetitive long-term administration during the first months of life to evaluate the durability of NRG1 in the preservation of RV function in children with CHD.

The most accurate way to assess the proliferation of cardiomyocytes is a matter of ongoing debate. ${ }^{15}$ We assessed Ki-67, a marker of cell cycle activity, which, during postnatal development, can be coupled to polyploidization in cardiomyocytes rather than cell division, as described in the proliferation consensus statement. ${ }^{11}$ In contrast to many other studies, we combined the staining with PCM-1, in the heart a specific marker for cardiomyocyte nuclei, ${ }^{9}$ to assess CCA. In addition, we used H3P, a marker of mitosis, to confirm this signal. Yet, H3P represents actual nucleus division, which is known to be present in low amounts. ${ }^{9,15}$ In line with this knowledge, the number of events appeared too low to detect differences. Also, our study did not find increased gene activity known to play a role in the pathways that stimulate cardiomyocyte proliferation through NRG1. ${ }^{35}$ Combined, these findings indicate cell cycle activation without progression to actual mitosis.

\section{Limitation}

The RNA expression profile was obtained in whole cardiac tissue, which is constituted of different cell types including endothelial cells, epicardial cells, macrophages, cardiomyocytes, and fibroblasts. Cell lineage tracing are not feasible in the rat at present, but development of single-cell sequencing might be used in further studies. The PL we applied in the present study was more progressive than that applied previously in adult rats. ${ }^{5}$ This is because of the growth of the rats with a fixed banding. This could be viewed as a strength of the study because we studied adaptation throughout growth and development.

\section{CONCLUSIONS}

RV PL applied in rats during postnatal growth and development transiently increased CCA. Stimulation of CCA via exogenous administration of NRG1 improved cardiac function, decreased fibrosis, and improved clinical outcome (Figure 6). The results of this proof-of-concept study imply that stimulation of CCA might provide a new approach to preserve RV function in patients with CHD.

\section{Conflict of Interest Statement}

The authors reported no conflicts of interest.

The Journal policy requires editors and reviewers to disclose conflicts of interest and to decline handling or reviewing manuscripts for which they may have a conflict of interest. The editors and reviewers of this article have no conflicts of interest.

The authors thank Annemieke van Oosten and Michel Weij for their assistance in the central animal facility and performing 

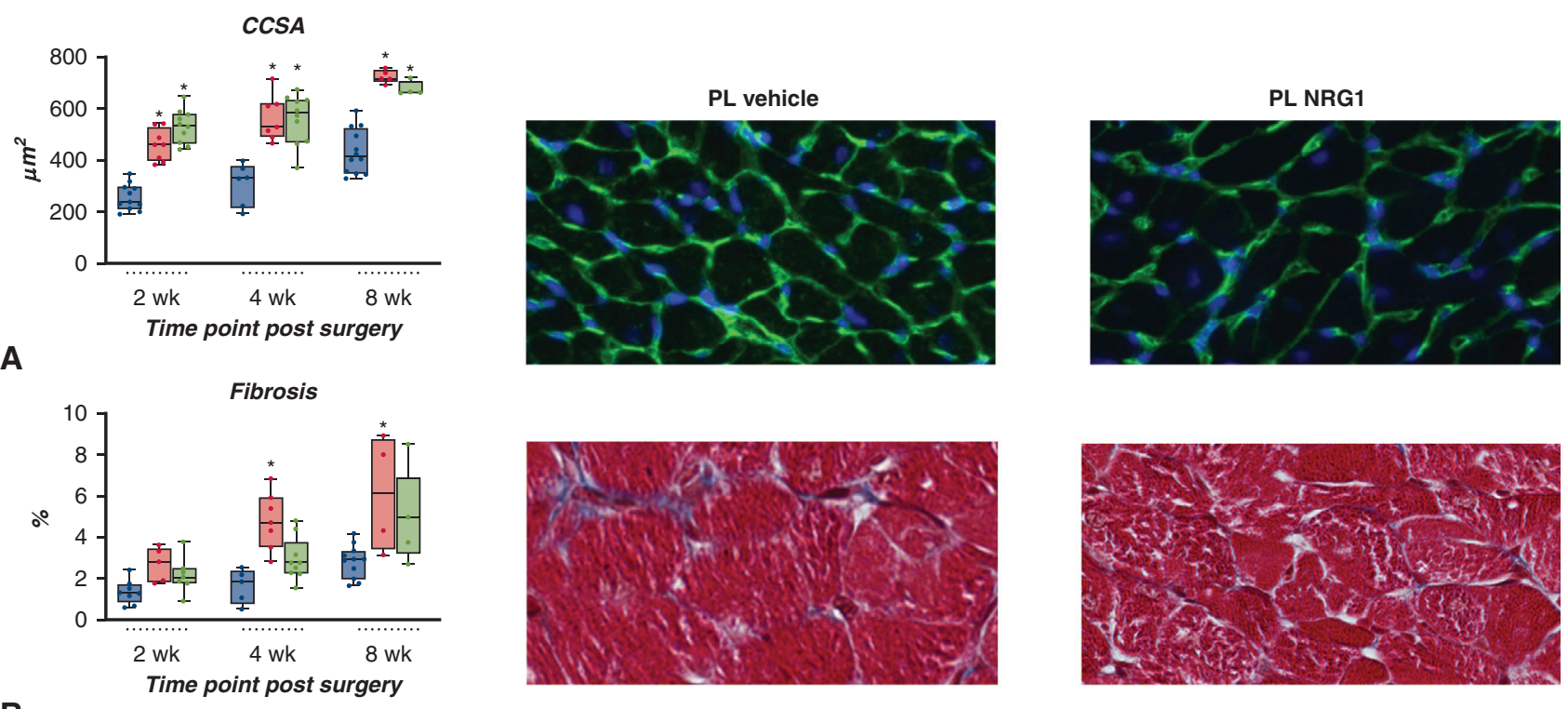

B
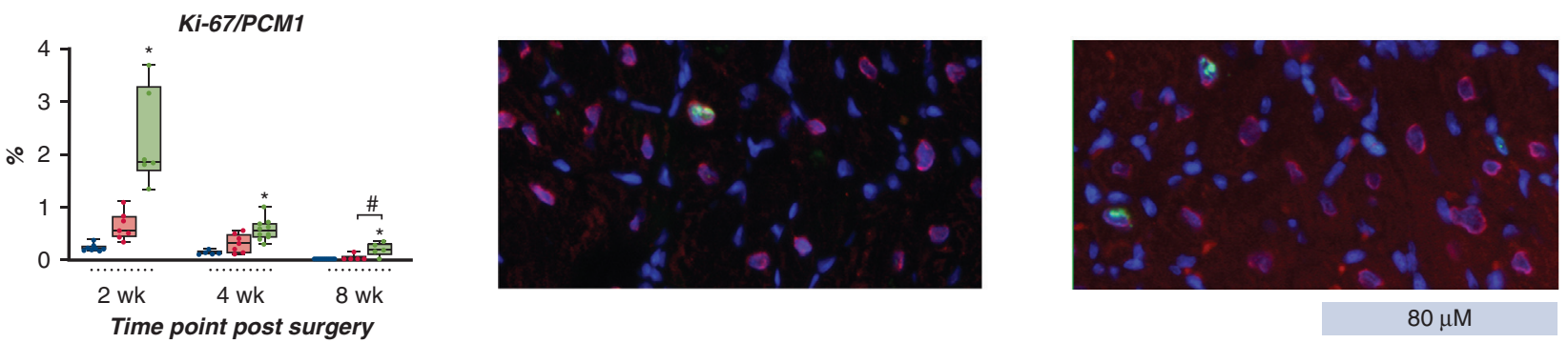

C

FIGURE 5. Histology and gene expression. A, No differences in right ventricular (RV) hypertrophy expressed in cardiomyocyte cross-sectional area $(C C S A)$ for neuregulin-1 (NRG1)- and vehicle-treated pressure load $(P L)$ rats (first row are representative images of wheat germ agglutinin-stained RVs 4 weeks after surgery). $P$ values: $2 / 4 / 8$ weeks control versus PL vehicle: $.012 / .003 / .003$; control versus PL NRG1: $<.001 /<.001 / .003 ; 2 / 4 / 8$ weeks PL vehicle versus PL NRG1: 1.000/1.000/.600. B, fibrosis is less pronounced in NRG1-treated PL rats (second row images are representative images of Masson-Trichrome-stained RVs 4 weeks after surgery). $P$ values: $2 / 4 / 8$ weeks control versus PL vehicle: .018/.000/.054; control versus PL NRG1: .162/.087/.114; 2/4/ 8 weeks PL vehicle versus PL NRG1: 1.000/.036/1.000. C, NRG1 increased cardiomyocyte specific cell cycle activity in PL rats (third row: representative slides of DAPI [blue]/Ki-67 (green)/pericentriolar material 1 [PCM-1], red) staining in the right ventricle at 2 weeks of control and PL right ventricle. $P$ values: 2/4/8 weeks control versus PL vehicle: .003/.219/1.000; control versus PL NRG1: .003/.039/.027; 2/4/8 weeks PL vehicle versus PL NRG1: .003/ .327/.189. D, mRNA expression of genes from left to right related to fibrosis, dedifferentiation/cell cycle activity, and metabolism. $P$ values: $2 / 4 / 8$ weeks control versus PL vehicle: $<.001 / .009 / .078$; control versus PL NRG1: $<.001 / .003 / .078 ;$; $/ 4 / 8$ weeks PL vehicle versus PL NRG1: .051/1.000/1.000. $P$ values: 2/4/8 weeks control versus PL vehicle: <.001/.015/.090; control versus PL NRG1: .012/.024/.819; 2/4/8 weeks PL vehicle versus PL NRG1: .399/1.000/1.000. $P$ values: $2 / 4 / 8$ weeks control versus PL vehicle: .066/.318/1.000; control versus PL NRG1: .840/.387/.309; $2 / 4 / 8$ weeks PL vehicle versus PL NRG1: 1.000/1.000/1.000. $P$ values: 2/4/8 weeks control versus PL vehicle: .024/.009/.078; control versus PL NRG1: .003/.015/.078; 2/4/8 weeks PL vehicle versus PL NRG1: 1.000/1.000/.999. $P$ values: $2 / 4 / 8$ weeks control versus PL vehicle: 1.000/.009/1.000; control versus PL NRG1: 1.000/.039/1.000; 2/4/8 weeks PL vehicle versus PL NRG1: 1.000/.483/.999. Red bars represent PL vehicle rats, green bars represent PL NRG1 rats, blue bars represent control rats. In some groups, numbers are lower than mentioned in methods because of failed analyses. The lower and upper borders of the box are the lower and upper quartiles. The middle horizontal line represents the median. The whiskers are the minimum and maximum values. Dots in the box-and-whisker dot plots represent individual measurements. Statistical analysis: Kruskal-Wallis with correction for multiple testing. Timp-1, Metallopeptidase inhibitor 1; Runx, runt-related transcription factor 1; Dab2, disabled adaptor protein 2; MCAD, medium-chain acyl-CoA dehydrogenase; Glut1, glucose transporter 1. ${ }^{*} P<.05$ compared with control. $\# P<.05$ PL vehicle compared with NRG1. 

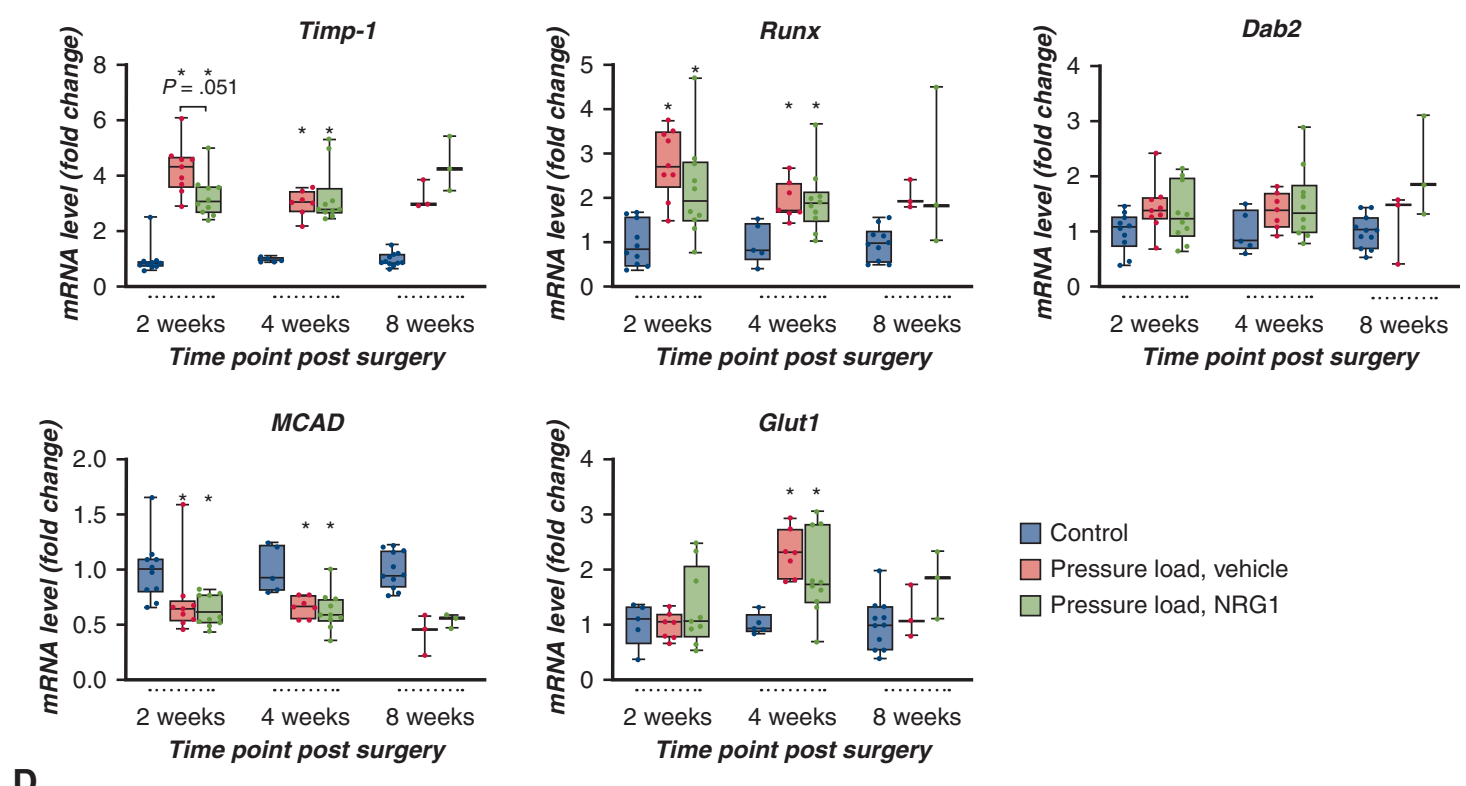

Control

$\square$ Pressure load, vehicle

$\square$ Pressure load, NRG1

FIGURE 5. Continued.

Methods

Pulmonary artery banding

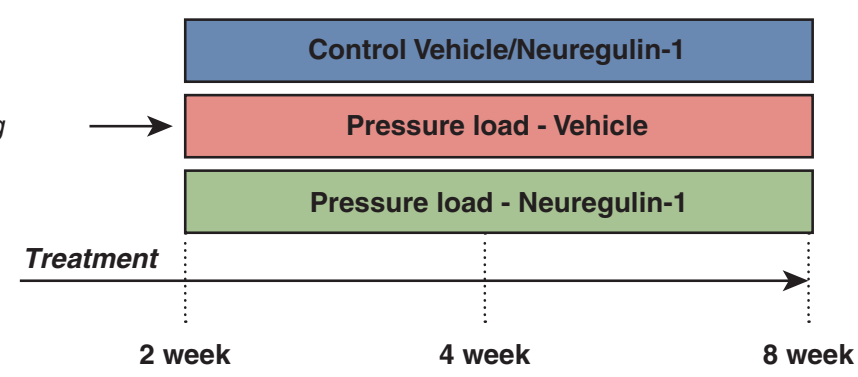

Results

Ki67/PCM1

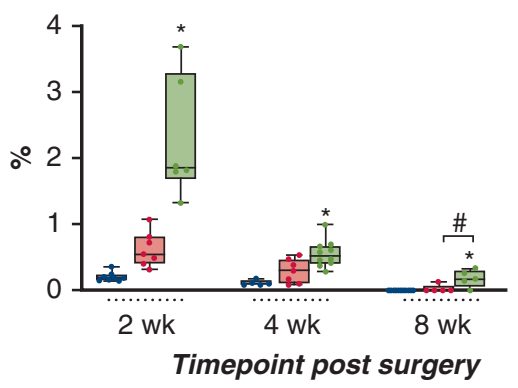

Cardiac index

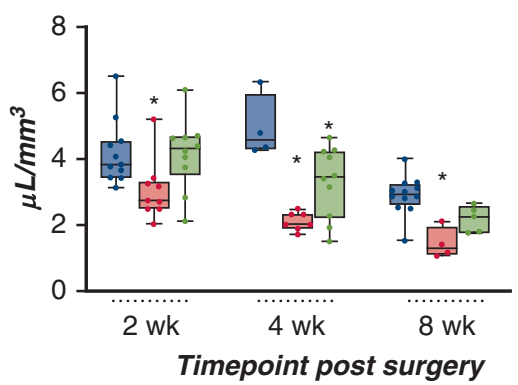

Fibrosis

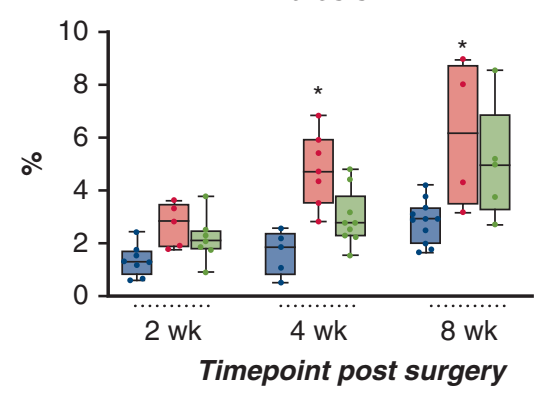

Implications

Stimulation with growth factors within window of regeneration may improve right ventricular function

FIGURE 6. Summary of the experiment. Red bars represent pressure load (PL) vehicle rats, green bars represent PL neuregulin-1 (NRG1) rats, blue bars represent control rats. Results: Ki-67/pericentriolar material 1 (PCM-1) $P$ values: 2/4/8 weeks control versus PL vehicle: .003/.219/1.000; control versus PL NRG1: .003/.039/.027; 2/4/8 weeks PL vehicle versus PL NRG1: .003/.327/.189. Cardiac index $P$ values: $2 / 4 / 8$ weeks control versus PL vehicle: .018/.000/ .054; control versus PL NRG1: .162/.087/.114; $2 / 4 / 8$ weeks PL vehicle versus PL NRG1: 1.000/.036/1.000. Fibrosis $P$ values: $2 / 4 / 8$ weeks control versus PL vehicle: .018/.000/.054; control versus PL NRG1: .162/.087/.114; 2/4/8 weeks PL vehicle versus PL NRG1: 1.000/.036/1.000. The lower and upper borders of the box are the lower and upper quartiles. The middle horizontal line represents the median. The whiskers are the minimum and maximum values. Dots in the box-and-whisker dot plots represent individual measurements. $* P<.05$ versus control. $\# P<.05$ NRG1 versus vehicle. 
pulmonary artery banding surgery. Special thanks to Tom van Leusden and Martin Dokter for their technical assistance.

\section{References}

1. Stout KK, Broberg CS, Book WM, Cecchin F, Chen J, Dimopoulos K, et al. Chronic heart failure in congenital heart disease. Circulation. 2016;133:770-801.

2. Triedman JK, Newburger JW. Trends in congenital heart disease. Circulation. 2016:133:2716-33.

3. Kuehl KS, Landzberg MJ, Mahle WT, Mann D, Marelli A, Newburger J, et al. Emerging research directions in adult congenital heart disease: a report from an NHLBI/ACHA working group. J Am Coll Cardiol. 2016; 67:1956-64.

4. Borgdorff MAJ, Dickinson MG, Berger RMF, Bartelds B. Right ventricular failure due to chronic pressure load: what have we learned in animal models since the NIH working group statement? Heart Fail Rev. 2015;20:475-91.

5. Borgdorff MAJ, Koop AMC, Bloks VW, Dickinson MG, Steendijk P, Sillje HHW, et al. Clinical symptoms of right ventricular failure in experimental chronic pressure load are associated with progressive diastolic dysfunction. J Mol Cell Cardiol. 2015;79:244-53.

6. van de Veerdonk MC, Bogaard HJ, Voelkel NF. The right ventricle and pulmonary hypertension. Heart Fail Rev. 2016;21:259-71.

7. Konstam MA, Kiernan MS, Bernstein D, Bozkurt B, Jacob M, Kapur NK, et al. Evaluation and management of right-sided heart failure: a scientific statement from the American Heart Association. Circulation. 2018;137:e578-622.

8. Khairy P, Ionescu-Ittu R, MacKie AS, Abrahamowicz M, Pilote L, Marelli AJ. Changing mortality in congenital heart disease. J Am Coll Cardiol. 2010;56: 1149-57.

9. Bergmann O, Zdunek S, Felker A, Salehpour M, Alkass K, Bernard S, et al. Dynamics of cell generation and turnover in the human heart. Cell. 2015;161: $1566-75$.

10. Mollova M, Bersell K, Walsh S, Savla J, Das LT, Park S, et al. Cardiomyocyte proliferation contributes to heart growth in young humans. Proc Natl Acad Sci U S A. 2013;110:1446-51.

11. Eschenhagen T, Bolli R, Braun T, Field L, Fleischmann BK, Frisén J, et al. Cardiomyocyte regeneration: a consensus statement. Circulation. 2017;8:680-6.

12. Wadugu B, Kuhn B. The role of neuregulin/ErbB2/ErbB4 signaling in the heart with special focus on effects on cardiomyocyte proliferation. Am J Physiol Heart Circ Physiol. 2012;302:H2139-47.

13. Sengupta P. The laboratory rat: relating its age with human's. Int J Prev Med. 2013;4:624-30

14. Hagdorn QAJ, Bossers GPL, Koop AMC, Piek A, Eijgenraam TR, van der Feen DE, et al. A novel method optimizing the normalization of cardiac parameters in small animal models: the importance of dimensional indexing. Am J Physiol Heart Circ Physiol. 2019;316:H1552-7.

15. Leone M, Magadum A, Engel FB. Cardiomyocyte proliferation in cardiac development and regeneration: a guide to methodologies and interpretations. Am J Physiol Heart Circ Physiol. 2015:309:H1237-50.

16. Bergmann O, Zdunek S, Alkass K, Druid H, Bernard S, Frisén J. Identification of cardiomyocyte nuclei and assessment of ploidy for the analysis of cell turnover. Exp Cell Res. 2011;317:188-94.

17. Ko JH, Eom GH, Cho HJ, Nam K, Ma JS, Kook H, et al. Right ventricular myocardial performance index is decreased with severe pressure-overload cardiac hypertrophy in young rats. Pediatr Cardiol. 2013;34:1556-66.

18. Ye L, Wang S, Xiao Y, Jiang C, Huang Y, Chen H, et al. Pressure overload greatly promotes neonatal right ventricular cardiomyocyte proliferation: a new model for the study of heart regeneration. J Am Heart Assoc. 2020;9:e015574.
19. Bond AR, Iacobazzi D, Abdul-Ghani S, Ghorbel M, Heesom K, Wilson M, et al Changes in contractile protein expression are linked to ventricular stiffness in infants with pulmonary hypertension or right ventricular hypertrophy due to congenital heart disease. Open Heart. 2018;5:1-14.

20. Zomer AC, Vaartjes I, Van Der Velde ET, De Jong HMY, Konings TC, Wagenaar LJ, et al. Heart failure admissions in adults with congenital heart disease; risk factors and prognosis. Int J Cardiol. 2013;168:2487-93.

21. Hillgaard TK, Andersen A, Andersen S, Vildbrad MD, Ringgaard S, Nielsen JM, et al. Levosimendan prevents pressure-overload-induced right ventricular failure. J Cardiovasc Pharmacol. 2016:67:275-82.

22. Porrello ER, Mahmoud AI, Simpson E, Hill JA, Richardson JA, Olson EN, et al Transient regenerative potential of the neonatal mouse heart. Science. 2011;331: 1078-81.

23. Pasumarthi KBS, Nakajima H, Nakajima HO, Soonpaa MH, Field LJ. Targeted expression of cyclin D2 results in cardiomyocyte DNA synthesis and infarct regression in transgenic mice. Circ Res. 2005;96:110-8.

24. Tian Y, Liu Y, Wang T, Zhou N, Kong J, Chen L, et al. A microRNA-Hippo pathway that promotes cardiomyocyte proliferation and cardiac regeneration in mice. Sci Transl Med. 2015;7:279ra38.

25. Nakada Y, Canseco DC, Thet S, Abdisalaam S, Asaithamby A, Santos CX, et al. Hypoxia induces heart regeneration in adult mice. Nature. 2017;541:222-7.

26. Bersell K, Arab S, Haring B, Kühn B. Neuregulin1/ErbB4 signaling induces cardiomyocyte proliferation and repair of heart injury. Cell. 2009;138:257-70

27. Polizzotti BD, Ganapathy B, Walsh S, Choudhury S, Ammanamanchi N Bennett DG, et al. Neuregulin stimulation of cardiomyocyte regeneration in mice and human myocardium reveals a therapeutic window. Sci Transl Med. 2015;7:281ra45

28. Kirabo A, Ryzhov S, Gupte M, Sengsayadeth S, Gumina RJ, Sawyer DB, et al Neuregulin- $1 \beta$ induces proliferation, survival and paracrine signaling in normal human cardiac ventricular fibroblasts. J Mol Cell Cardiol. 2017:105:59-69.

29. Adão R, Mendes-Ferreira P, Maia-Rocha C, Santos-Ribeiro D, Rodrigues SG, Vidal-Meireles A, et al. Neuregulin-1 attenuates right ventricular diastolic stiffness in experimental pulmonary hypertension. Clin Exp Pharmacol Physiol. 2019;46: 255-65.

30. Mendes-Ferreira P, Maia-Rocha C, Adão R, Mendes MJ, Santos-Ribeiro D, Alves BS, et al. Neuregulin-1 improves right ventricular function and attenuates experimental pulmonary arterial hypertension. Cardiovasc Res. 2016;109:44-54.

31. Crnkovic S, Egemnazarov B, Damico R, Marsh LM, Nagy BM, Douschan P, et al. Disconnect between fibrotic response and right ventricular dysfunction. Am J Respir Crit Care Med. 2019;199:1550-60.

32. Ganapathy B, Nandhagopal N, Polizzotti BD, Bennett D, Asan A, Wu Y, et al Neuregulin-1 administration protocols sufficient for stimulating cardiac regeneration in young mice do not induce somatic, organ, or neoplastic growth. PLoS One. 2016;11:1-20.

33. Haller C, Friedberg MK, Laflamme MA. The role of regenerative therapy in the treatment of right ventricular failure: a literature review. Stem Cell Res Ther 2020;11:1-9.

34. Mendes-Ferreira P, De Keulenaer GW, Leite-Moreira AF, Brás-Silva C. Therapeutic potential of neuregulin-1 in cardiovascular disease. Drug Discov Today. 2013;18:836-42

35. D’Uva G, Aharonov A, Lauriola M, Kain D, Yahalom-Ronen Y, Carvalho S, et al ERBB2 triggers mammalian heart regeneration by promoting cardiomyocyte dedifferentiation and proliferation. Nat Cell Biol. 2015;17:627-38.

Key Words: right ventricle, proliferation, heart failure, pressure load, neuregulin-1 


\section{APPENDIX E1. METHODS \\ RNA Sequencing}

For RNA sequencing analysis, samples were used of rats that were analyzed for the characterization study (time point 4 and 8 weeks after surgery). RNA was extracted from the right ventricular free wall, in 3 biological replicates of $800 \mathrm{ng}$ each. After quality control, the RNAseq library was prepared using the TruSeq Ribo Gold kit (Illumina). The library was sequenced on 2 HiSeq4000 lanes (Illumina). Adapter and polyA sequences and low-quality nucleotides were removed using BB Tools Decontamination Using Kmers (BBDuk). Trimmed reads were mapped against the Rnor_6.0 rat genome using Spliced Transcripts Alignment to a Reference (STAR), and high-throughput sequence analysis count (htseq-count) was used to determine read counts. After quality control with fast quality control (FastQC), biological replicates were averaged, followed by differential expression analysis using the DESeq2 package (PMID: 25516281). Differentially expressed genes were included at cutoffs of $>1 \log 2$-fold change and false discovery rate (FDR)-adjusted $P$ value $<.05$. Kyoto Encyclopedia of Genes and Genomes (KEGG) pathway and gene ontology analyses were conducted using Database for Annotation, Visualization, and Integrated Discovery (DAVID).

Principal component analysis was performed on rlognormalized RNAseq data using the DESeq2 package. The first 2 principle components (PCs), $\mathrm{PC} 1$ and $\mathrm{PC} 2$, are reported in the graph on $\mathrm{x}$ - and $\mathrm{y}$-axes, respectively (Figure $\mathrm{E} 1, A)$.

Differential expression analysis was performed using the DESeq2 package. $P$ values were corrected for multiple testing using the FDR method of Benjamini-Hochberg (FDR < 0.05). 


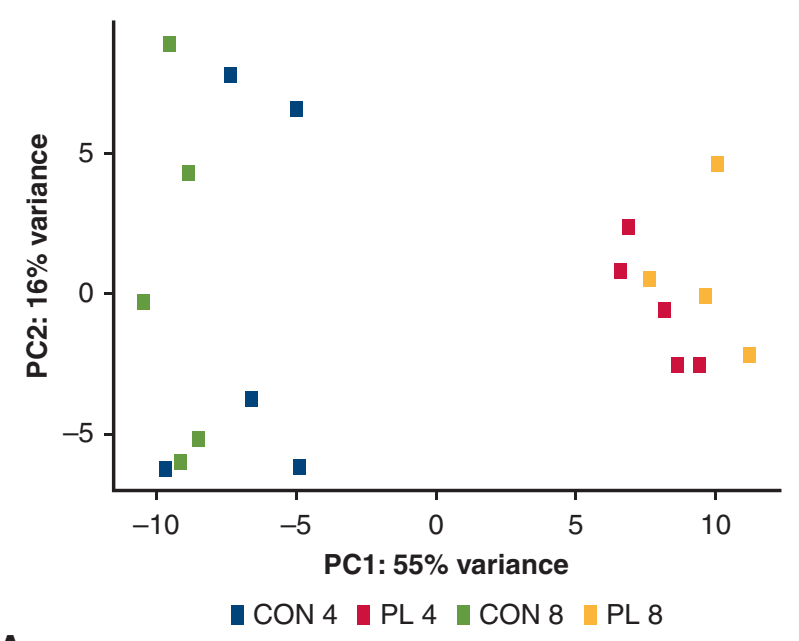

A

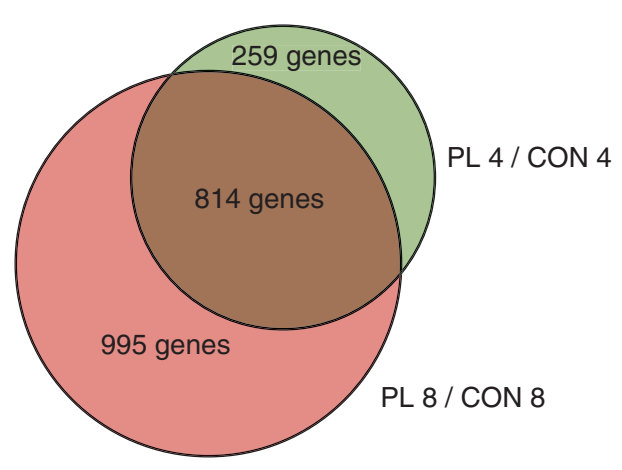

GO Term

$\underline{P \text { value }}$

1. Cell division

$7.80 e^{-04}$

3. Endosome

$7.70 \mathrm{e}^{-03}$

1. Cell-cell adhesion $\quad 2.80 \mathrm{e}^{-07}$

2. Stress response $\quad 1.00 \mathrm{e}^{-02}$

3. Nucleotide-binding $\quad 9.00 \mathrm{e}^{-07}$

4. EGF-like domain $\quad 1.70 \mathrm{e}^{-04}$

1. Immunity $\quad 9.20 \mathrm{e}^{-11}$

2. Response to virus $\quad 3.70 \mathrm{e}^{-05}$

3. Pleckstrin homology-like domain $2.50 \mathrm{e}^{-05}$

4. cell-cell adherens junction $\quad 6.20 \mathrm{e}^{-04}$

5. Glycoportein $\quad 6.70 \mathrm{e}^{-09}$

\section{B}

FIGURE E1. A, Principal component $(P C)$ analysis clustered the right ventricle (RV) by pressure load $(P L)$. B, Comparison of differentially expressed genes in PL versus control $(C O N)$ between 4 and 8 weeks identified genes involved in cell division to be involved at 4 weeks. Statistical analysis: see paragraph "RNA Sequencing" in Appendix E1. GO, Gene ontology; EGF, epidermal growth factor.

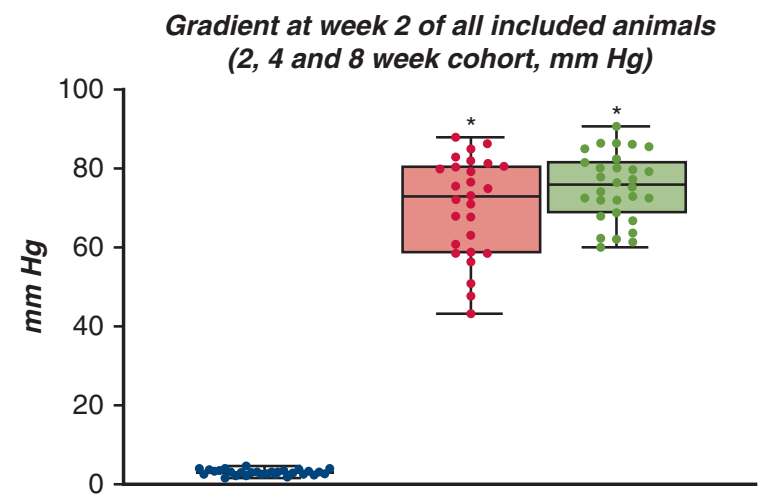

$\square$ Control $\square$ Pressure load vehicle $\square$ Pressure load NRG1

FIGURE E2. Gradient at week 2 of all included animals. Red bars represent pressure load (PL) vehicle rats, green bars represent PL neuregulin-1 $(N R G 1)$ rats, blue bars represent control rats. $P$ values: week control versus PL vehicle: $<.001$; control versus PL NRG1: $<.001$; PL vehicle versus PL NRG1: .468. The lower and upper borders of the box are the lower and upper quartiles. The middle horizontal line represents the median. The whiskers are the minimum and maximum values. Dots in the box-and-whisker dot plots represent individual measurements. ${ }^{*} P<.05$ compared with control. 
TABLE E1. Sequence of primers used for quantitative real-time polymerase chain reaction

\begin{tabular}{|c|c|c|}
\hline & Sequence forward primer & Sequence reverse primer \\
\hline GADPH & TCTCTGCTCCTCCCTGTTCTA & TACGGCCAAATCCGTTCACA \\
\hline Nppa & ATGGGCTCCTTCTCCATCAC & TCTACCGGCATCTTCTCCTC \\
\hline Nppb & СТССТАTССТCTGGGAAGTC & CCGATCCGGTCTATCTTGTG \\
\hline Timp-1 & AGAGCCTCTGTGGATATGTC & CTCAGATTATGCCAGGGAAC \\
\hline HO-1 & CAGAGGGTGACAGAAGAGGCTAA & CTGGTCTTTGTGTTCCTCTGTCAG \\
\hline MCAD & CCGTTCССТCTCATCAAAAG & ACACCCATACGCCAACTCTT \\
\hline Glut1 & GCTTTGGCAGGCGGAACT & AGCACGGCAGACACAAAGG \\
\hline Runx1 & AACCCTCAGCCTCAAAGTCA & GGGTGCACAGAAGAGGTGAT \\
\hline Dab2 & CTCAGCGCATGACTCCATAG & CTGAAGACTTAGCGGTCCTC \\
\hline Myh7 & ATCCTCATCACCGGAGAATC & CCCAATGGCAGCAATAACAG \\
\hline Myh6 & GCGTGTCATCCAGTACTTTG & GGTGCCCTTGTTTGCATTAG \\
\hline
\end{tabular}

